\title{
A TRUST-REGION ALGORITHM WITH ADAPTIVE STOCHASTIC COLLOCATION FOR PDE OPTIMIZATION UNDER UNCERTAINTY*
}

\author{
D. P. KOURI ${ }^{\dagger}$, M. HEINKENSCHLOSS $\ddagger$, D. RIDZAL ${ }^{\S}$, AND \\ B. G. VAN BLOEMEN WAANDERS 『
}

\begin{abstract}
The numerical solution of optimization problems governed by partial differential equations (PDEs) with random coefficients is computationally challenging because of the large number of deterministic PDE solves required at each optimization iteration. This paper introduces an efficient algorithm for solving such problems based on a combination of adaptive sparse-grid collocation for the discretization of the PDE in the stochastic space and a trust-region framework for optimization and fidelity management of the stochastic discretization. The overall algorithm adapts the collocation points based on the progress of the optimization algorithm and the impact of the random variables on the solution of the optimization problem. It frequently uses few collocation points initially and increases the number of collocation points only as necessary, thereby keeping the number of deterministic PDE solves low while guaranteeing convergence. Currently an error indicator is used to estimate gradient errors due to adaptive stochastic collocation. The algorithm is applied to three examples, and the numerical results demonstrate a significant reduction in the total number of PDE solves required to obtain an optimal solution when compared with a Newton conjugate gradient algorithm applied to a fixed high-fidelity discretization of the optimization problem.
\end{abstract}

Key words. PDE optimization, uncertainty, stochastic collocation, trust regions, sparse grids, adaptivity

AMS subject classifications. 49M15, 65K05, 65N35, 90C15

DOI. $10.1137 / 120892362$

1. Introduction. Optimization problems governed by partial differential equations (PDEs) arise in many important science and engineering applications. In most applications model parameters, such as diffusivity in a heat equation, or rates in reaction equations are not known exactly and have to be modeled as random variables or random fields. It is important to include the randomness of the data in the formulation of the optimization problem. However, the numerical solution of the resulting optimization problem is expensive, since one not only needs to discretize the governing PDE in space (and time) but also needs to approximate the random variables. In this

*Submitted to the journal's Methods and Algorithms for Scientific Computing section September 24, 2012; accepted for publication (in revised form) May 6, 2013; published electronically July 9, 2013. Sandia National Laboratories is a multi-program laboratory managed and operated by Sandia Corporation, a wholly owned subsidiary of Lockheed Martin Corporation, for the U.S. Department of Energy's National Nuclear Security Administration under contract DE-AC04-94AL85000.

http://www.siam.org/journals/sisc/35-4/89236.html

${ }^{\dagger}$ Mathematics and Computer Science Division, Argonne National Laboratory, Argonne, IL 604394844 (dpkouri@mcs.anl.gov). This author's work was supported in part by AFOSR grant FA9550-091-0225, by NSF grant DMS-0915238, and by the Office of Advanced Scientific Computing Research, Office of Science, U.S. Department of Energy, under contract DE-AC02-06CH11357.

¥Department of Computational and Applied Mathematics, Rice University, Houston, TX 770051892 (heinken@rice.edu). This author's work was supported in part by AFOSR grants FA9550-091-0225 and FA9550-12-1-0155 and by NSF grant DMS-0915238.

$\S$ Optimization and Uncertainty Quantification, Sandia National Laboratories, Albuquerque, NM 87185-1320 (dridzal@sandia.gov). This author's research was sponsored by the NNSA Advanced Scientific Computing (ASC) program.

ॠNumerical Analysis and Applications, Sandia National Laboratories, Albuquerque, NM 871851320 (bartv@sandia.gov). This author's research was sponsored by the NNSA Advanced Scientific Computing (ASC) program. 
paper we consider optimization problems governed by elliptic PDEs with random data and deterministic optimization (control) variables. This formulation of the optimization problem arises when one must decide on the control action prior to observing the outcome.

The main focus of this paper is on the efficient numerical solution of the PDE constrained optimization problem with random data and deterministic optimization (control) variables. We begin by presenting a framework for stating the infinitedimensional problem. However, our main contribution is the introduction and analysis of an optimization algorithm based on a combination of adaptive sparse-grid collocation for the discretization of the PDE in the stochastic space and a trust-region framework for optimizing and managing the fidelity of the discretization.

Our overall algorithm adapts the collocation points based on the progress of the optimization algorithm and the impact of the random variables on the solution of the optimization problem. In our numerical examples, our algorithm uses few collocation points initially and increases the number of collocation points only as necessary, thereby keeping the number of deterministic PDE solves low while guaranteeing convergence, provided the error due to adaptive stochastic collocation can be estimated.

Numerical methods for solving PDEs with random data can be classified as projection-based methods and sample-based methods. Common projection-based methods are polynomial chaos and stochastic Galerkin methods [4, 5, 37, 67]; common sampling methods are Monte Carlo and stochastic collocation [3, 47, 46, 66]. The algorithm presented in this paper is based on stochastic collocation. This allows us to exploit the problem structure that arises from sampling methods. Moreover, stochastic collocation is an interpolation-based technique that exhibits faster convergence than Monte Carlo methods when the random-field solution of the PDE is sufficiently regular with respect to the random variables $[3,47,46]$.

The stochastic collocation discretization scheme with fixed collocation level is analyzed for PDE-constrained optimization problems in [39]. In this paper we adapt the stochastic collocation. More specifically, we minimize a fixed high-fidelity collocation approximation of the infinite-dimensional objective function. In each optimization iteration we use adapted collocation to model the high-fidelity objective function. The models typically have a significantly smaller number of collocation points. The trust-region framework [2] is used to specify the model fidelity required at each iteration. We use conditions derived from [33] to specify the model fidelity needed to ensure convergence of the trust-region method to a point that satisfies the first-order optimality conditions.

To derive models that meet the accuracy requirements specified by our trustregion method, we use the structure of the collocation discretization, and we employ sparse grids $[58,25,48,49,6,64,14,50,51,52]$. Specifically, we use stochastic collocation built on dimension-adaptive sparse grids [26]. This approximation provides an error indicator, not an error estimate, which is required by our trustregion convergence theory. This heuristic approach performs well in our numerical tests.

After discretization, our optimization problem is a particular finite-dimensional stochastic programming problem. Such problems are discussed, for example, in [57]. Since our optimization problems involve PDEs, their efficient solution requires additional considerations. The integration of adaptive sparse grids and trust-region methods is new, as is the application to several PDE constrained problems. We mention that the authors of [7] combine a trust-region framework and Monte Carlo sampling 
for the solution of a class of stochastic programming problems, the so-called mixed logit problem.

Optimization problems governed by PDEs with random data are studied in, for example, $[12,13,16,22,23,53,24,30,39,40,55,56,60]$. Unlike our case, where the optimization variable is deterministic, the papers $[40,13,60]$ study problems where the optimization variable is also a random field. This approach impacts the structure of the optimization problems. However, it seems possible to extend our numerical approach to those problems. The papers [55, 56, 16, 24, 53] focus on specific applications, including aerodynamic design under uncertainty, optimization of radiated engine noise, optimal control of stochastic heating processes, and optimal control of turbulence, respectively. It is possible to apply our solution approach to several of the problems (see also section 5). The authors of [55, 56, 60] employ sparse-grid collocation but do not provide a convergence analysis of their approach. The papers $[30,39]$ provide a discretization error analysis for stochastic Galerkin and stochastic collocation approximations, respectively. Our paper integrates adaptive sparse grids and trust-region methods and provides a convergence analysis, which is new. We also analyze the application of our method to several PDE-constrained problems.

Our paper is organized as follows. First, we present a problem formulation for the class of optimization problems we consider. We state assumptions that guarantee wellposedness of the optimization problems and allow gradient computations. Section 3 reviews sparse grids and the dimension-adaptive sparse grids of [26]. Most of the material in this section is known, but it is presented in the context of optimization so that it can be integrated with our optimization algorithm. In section 4, we present the trust-region algorithm and describe how we construct models using adaptive sparse grids. The presentation of the trust-region method is for more general optimization problems. We use conditions derived from [33] to specify the model fidelity needed to ensure convergence of the trust-region method to a point that satisfies the first-order optimality conditions. In section 5, we apply our approach to three examples. In particular, we show how these examples fit into the abstract problem statement of section 2, and we illustrate the computational savings achieved by our integration of adaptive sparse grids and trust-region based optimization.

2. Problem formulation. Let $(\Omega, \mathcal{F}, P)$ denote a complete probability space, where $\Omega$ is the set of outcomes, $\mathcal{F}$ is the $\sigma$-algebra of events, and $P: \mathcal{F} \rightarrow[0,1]$ is a probability measure. Furthermore, let $\mathcal{V}$ and $\mathcal{Z}$ denote real Hilbert spaces. For almost every $\omega \in \Omega$, let $\mathbf{A}(\omega) \in \mathcal{L}\left(\mathcal{V}, \mathcal{V}^{*}\right), \mathbf{B}(\omega) \in \mathcal{L}\left(\mathcal{Z}, \mathcal{V}^{*}\right), \mathbf{b}(\omega) \in \mathcal{V}^{*}$, and $\mathbf{N}(\cdot, \omega)$ : $\mathcal{V} \rightarrow \mathcal{V}^{*}$. We will consider optimization problems governed by the stochastic equation

$$
\mathbf{A}(\omega) u(\omega)+\mathbf{N}(u(\omega), \omega)+\mathbf{B}(\omega) z+\mathbf{b}(\omega)=0 \quad \text { a.e. in } \Omega .
$$

Here $u$ denotes the state variable, and $z$ denotes the control variable. The state $u$ is a random field, but the control is deterministic. This models the situation where one must decide on the control action prior to observing the outcome.

To facilitate the numerical solution of (2.1), we will work under the finite noise assumption; see [3].

Assumption 2.1. There exists a vector of random variables $Y: \Omega \rightarrow \Gamma \subset \mathbb{R}^{M}$ such that $\mathbf{A}(\omega) \equiv \mathbf{A}(Y(\omega)), \mathbf{B}(\omega) \equiv \mathbf{B}(Y(\omega)), \mathbf{b}(\omega) \equiv \mathbf{b}(Y(\omega))$, and $\mathbf{N}(\cdot, \omega) \equiv \mathbf{N}(\cdot, Y(\omega))$, where the components of $Y$ are independent random variables, $Y_{i}: \Omega \rightarrow \Gamma_{i} \subset \mathbb{R}$, with Lebesgue density $\rho_{i}: \Gamma \rightarrow \mathbb{R}$. The image space of $Y$ is $\Gamma=\prod_{i=1}^{M} \Gamma_{i}$ and the joint density of $Y$ is $\rho=\prod_{i=1}^{M} \rho_{i}$. 
The finite noise assumption allows the change of variables in which (2.1) is replaced by the parametrized system

$$
\mathbf{A}(y) u(y)+\mathbf{N}(u(y), y)+\mathbf{B}(y) z+\mathbf{b}(y)=0 \quad \forall y \in \Gamma .
$$

Our solution space for the state equation is the Bochner space $C_{\rho}^{0}(\Gamma ; \mathcal{V})$. Given a Banach space $\mathcal{X}$, the Bochner spaces used in this paper are

$$
\begin{aligned}
& L_{\rho}^{q}(\Gamma ; \mathcal{X})=\left\{u: \Gamma \rightarrow \mathcal{X}: u \text { strongly measurable, } \int_{\Gamma} \rho\|u\|_{\mathcal{X}}^{q}<\infty\right\} \text { for } q \in[1, \infty), \\
& C_{\rho}^{0}(\Gamma ; \mathcal{X})=\left\{u: \Gamma \rightarrow \mathcal{X}: u \text { is continuous and } \sup _{y \in \Gamma}\|\rho(y) u(y)\|_{\mathcal{X}}<\infty\right\} .
\end{aligned}
$$

The space $L_{\rho}^{q}(\Gamma ; \mathcal{X})$ and $C_{\rho}^{0}(\Gamma ; \mathcal{X})$ are Banach spaces, and $L_{\rho}^{2}(\Gamma ; \mathcal{V})$ is a Hilbert space with inner product $\langle u, v\rangle_{L_{\rho}^{2}(\Gamma ; \mathcal{V})}=\int_{\Gamma} \rho(y)\langle u(y), v(y)\rangle_{\mathcal{V}} \mathrm{d} y$. Furthermore, if $\mathcal{X}$ is reflexive and if $q, q^{*} \in(1, \infty)$ are conjugate pairs (i.e., $1 / q+1 / q^{*}=1$ ), then $\left(L_{\rho}^{q}(\Gamma ; \mathcal{X})\right)^{*}=L_{\rho}^{q^{*}}\left(\Gamma ; \mathcal{X}^{*}\right)$; see, for example, $[18,69,70]$. As usual, if $\mathcal{X}=\mathbb{R}$, we use the notation $L_{\rho}^{q}(\Gamma)$ and $C_{\rho}^{0}(\Gamma)$ instead of $L_{\rho}^{q}(\Gamma ; \mathbb{R})$ and $C_{\rho}^{0}(\Gamma ; \mathbb{R})$, respectively. We also note that $C_{1}^{0}(\Gamma ; \mathcal{X}) \subset L_{\rho}^{q_{1}}(\Gamma ; \mathcal{X}) \subset L_{\rho}^{q_{2}}(\Gamma ; \mathcal{X})$ for all $q_{1} \geq q_{2} \geq 1$ and, if $\Gamma$ is bounded, $C_{\rho}^{0}(\Gamma ; \mathcal{X}) \subset L_{\rho}^{q}(\Gamma ; \mathcal{X})$ for all $q \in[1, \infty)$.

In addition to the finite noise assumption, we will make the following assumption concerning the solvability of the state equation (2.2).

Assumption 2.2. For every $z \in \mathcal{Z}$ the state equation (2.2) has a unique solution $u(\cdot ; z) \in C_{\rho}^{0}(\Gamma ; \mathcal{V})$.

To formulate the optimization problem, we let $\mathcal{W}$ denote a real Hilbert space, $\mathbf{C} \in \mathcal{L}(\mathcal{V}, \mathcal{W})$, and $\bar{w} \in \mathcal{W}$. We consider an optimization problem whose objective function includes the term

$$
E\left[\|\mathbf{C} u(\cdot ; z)-\bar{w}\|_{\mathcal{W}}^{2}\right]=\int_{\Gamma} \rho(y)\|\mathbf{C} u(y ; z)-\bar{w}\|_{\mathcal{W}}^{2} d y .
$$

To ensure this objective function is well defined under Assumption 2.2, we require that $C_{\rho}^{0}(\Gamma ; \mathcal{V})$ be continuously embedded in $L_{\rho}^{2}(\Gamma ; \mathcal{V})$.

Assumption 2.3. The inclusion $C_{\rho}^{0}(\Gamma ; \mathcal{V}) \subset L_{\rho}^{2}(\Gamma ; \mathcal{V})$ holds, and there exists a constant $c>0$ such that $\|v\|_{L_{\rho}^{p}(\Gamma ; \mathcal{V})} \leq c\|v\|_{C_{\rho}^{0}(\Gamma ; \mathcal{V})}$ for all $v \in C_{\rho}^{0}(\Gamma ; \mathcal{V})$.

Given $\alpha>0$, we consider the optimization problem

$$
\min _{z \in \mathcal{Z}} J(z) \stackrel{\text { def }}{=} \frac{1}{2} E\left[\|\mathbf{C} u(\cdot ; z)-\bar{w}\|_{\mathcal{W}}^{2}\right]+\frac{\alpha}{2}\|z\|_{\mathcal{Z}}^{2},
$$

where $u(y ; z) \in \mathcal{V}$ for all $y \in \Gamma$ solves $(2.2)$. Such optimization problems are considered in, for example, $[12,56]$ and in the context of shape optimization in [23]. As we have mentioned, optimization problems of the form (2.3) arise when one must decide on the control action prior to observing the outcome. In contrast, papers $[11,13,60]$ consider optimization problems where the control $z$ also is a random field.

In many cases, the infinite-dimensional optimization problem can also be studied by using the weak form

$$
\int_{\Gamma} \rho(y)\langle\mathbf{A}(y) u(y)+\mathbf{N}(u(y), y)+\mathbf{B}(y) z+\mathbf{b}(y), \phi(y)\rangle_{\mathcal{V}^{*}, \mathcal{V}} d y=0 \quad \forall \phi \in L^{2}(\Gamma, \mathcal{V})
$$

and the state space $u(\cdot ; z) \in L_{\rho}^{p}(\Gamma ; \mathcal{V})$ for suitable $p \geq 2$. Since we will apply a stochastic collocation discretization, we consider $(2.2)$ and require $u(\cdot ; z) \in C_{\rho}^{0}(\Gamma ; \mathcal{V})$. 
In order to analyze convergence of the stochastic collocation discretization, additional smoothness of the state solution with respect to the parameters is required [39]. We can admit objective functions other than the expected value function, provided the state space is adjusted accordingly [39].

THEOREM 2.4. If Assumption 2.2 is satisfied and if for every sequence $\left\{z_{n}\right\}$ with $z_{n} \rightarrow z$ in $\mathcal{Z}$ the corresponding sequence $\left\{u\left(\cdot ; z_{n}\right)\right\}$ of states satisfies $u\left(\cdot ; z_{n}\right) \rightarrow u(\cdot ; z)$ in $L_{\rho}^{2}(\Gamma ; \mathcal{V})$, then the optimization problem $(2.3)$ has a solution.

Proof. This existence proof follows using standard arguments. See, for example, [36, sect. 1.5.1], [61, sect. 2.5].

The Fréchet differentiability of the objective function (2.3) can be established under the following condition.

Assumption 2.5. For every $y \in \Gamma$, the function $\mathcal{V} \ni u \mapsto \mathbf{N}(u, y) \in \mathcal{V}^{*}$ is Fréchet differentiable and the Fréchet derivative is denoted by $\mathbf{N}^{\prime}(u, y)$. Moreover, the function $\mathcal{Z} \ni z \mapsto u(\cdot ; z) \in C_{\rho}^{0}(\Gamma ; \mathcal{V})$ is Fréchet differentiable, and the derivative $v=u^{\prime}(\cdot ; z) \delta z$ satisfies

$$
\mathbf{A}(y) v(y)+\mathbf{N}^{\prime}(u(y), y) v(y)+\mathbf{B}(y) \delta z=0 \quad \forall y \in \Gamma .
$$

In addition, the adjoint equation

$$
\mathbf{A}(y)^{*} p(y)+\mathbf{N}^{\prime}(u(y), y)^{*} p(y)=-\mathbf{C}^{*}(\mathbf{C} u(y ; z)-\bar{w}) \quad \forall y \in \Gamma
$$

has a unique solution $p \in C_{\rho}^{0}(\Gamma ; \mathcal{V})$.

If Assumptions 2.2, 2.3, and 2.5 hold, the objective function (2.3) is Fréchet differentiable, and the Fréchet derivative is given by

$$
\begin{aligned}
J^{\prime}(z) \delta z & =\int_{\Gamma} \rho(y)\left\langle v(y), \mathbf{C}^{*}(\mathbf{C} u(z)-\bar{w})\right\rangle_{\mathcal{V}, \mathcal{V}^{*}} d y+\alpha\langle z, \delta z\rangle_{\mathcal{Z}} \\
& =-\int_{\Gamma} \rho(y)\left\langle v(y), \mathbf{A}(y)^{*} p(y)+\mathbf{N}^{\prime}(u(y), y)^{*} p(y)\right\rangle_{\mathcal{V}, \mathcal{V}^{*}}+\alpha\langle z, \delta z\rangle_{\mathcal{Z}} \\
& =-\int_{\Gamma} \rho(y)\left\langle\mathbf{A}(y) v(y)+\mathbf{N}^{\prime}(u(y), y) v(y), p(y)\right\rangle_{\mathcal{V}^{*}, \mathcal{V}}+\alpha\langle z, \delta z\rangle_{\mathcal{Z}} \\
& =\int_{\Gamma} \rho(y)\langle\mathbf{B}(y) \delta z, p(y)\rangle_{\mathcal{V}^{*}, \mathcal{V}}+\alpha\langle z, \delta z\rangle_{\mathcal{Z}} \\
& =\int_{\Gamma} \rho(y)\left\langle\mathbf{B}(y)^{*} p(y), \delta z\right\rangle_{\mathcal{Z}}+\alpha\langle z, \delta z\rangle_{\mathcal{Z}} .
\end{aligned}
$$

Thus, the gradient of the objective function $J(z)$ in $(2.3)$ is

$$
\nabla J(z)=\alpha z+\int_{\Gamma} \rho(y) \mathbf{B}(y)^{*} p(y) \mathrm{d} y=\alpha z+E\left[\mathbf{B}^{*} p\right] .
$$

Under additional assumptions, we can also compute second derivatives of the objective function $J(z)$ in (2.3). Moreover, standard first-order necessary optimality conditions and second-order necessary and sufficient optimality conditions can be stated. See, for example, [36, sect. 1.6].

3. Stochastic collocation. To discretize the optimal control problem (2.2), (2.3) we use stochastic collocation. For parametrized PDEs, such as (2.2), stochastic collocation has been extensively studied in, for example, [45, 47, 65, 66, 3], and stochastic collocation has been used in the optimization context in, for example, 
$[11,12,13,39,56]$. A discretization error analysis of stochastic collocation in the optimization context is provided in [39]. Stochastic collocation discretizations are attractive because they lead to decoupled systems of deterministic PDEs, which are easily parallelizable when implemented on a computer. Moreover, even when collocation points are chosen adaptively, which is our goal, the discretized objective function and its derivative are easy to evaluate.

In this section, we review the sparse-grid collocation method and an approach for the adaptive choice of collocation points. In the next section, we will integrate this material with a trust-region method in order to adaptively adjust the number of collocation points during optimization.

3.1. Stochastic collocation for PDEs. Let $\left\{y_{k}\right\}_{k=1}^{Q} \subset \Gamma$ be a finite set of points in $\Gamma$, and let $\left\{P_{k}\right\}_{k=1}^{Q} \subset C_{\rho}^{0}(\Gamma)$ be given polynomials. The stochastic collocation method approximates the solution $u \in C_{\rho}^{0}(\Gamma ; \mathcal{V})$ of $(2.2)$ as

$$
u_{Q}(y, x)=\sum_{k=1}^{Q} u_{k}(x) P_{k}(y),
$$

where $u_{k} \in \mathcal{V}$ solves

$$
\mathbf{A}\left(y_{k}\right) u_{k}+\mathbf{N}\left(u_{k}, y_{k}\right)+\mathbf{B}\left(y_{k}\right) z+\mathbf{b}\left(y_{k}\right)=0, \quad k=1, \ldots, Q .
$$

The choice of $\left\{y_{k}\right\}_{k=1}^{Q}$ and $\left\{P_{k}\right\}_{k=1}^{Q}$ governs the accuracy and efficiency of this algorithm $[45,47,65,3]$. We will use sparse-grid knots $\left\{y_{k}\right\}_{k=1}^{Q}$ and sparse-grid polynomials $\left\{P_{k}\right\}_{k=1}^{Q}$.

3.2. Stochastic collocation for optimization. Stochastic collocation can be used in different ways in the optimization context. See [39]. We discretize the objective function as

$$
\begin{aligned}
J_{Q}(z) & \stackrel{\text { def }}{=} \frac{1}{2} E\left[\sum_{k=1}^{Q} P_{k}\left\|\mathbf{C} u_{k}(z)-\bar{w}\right\|_{\mathcal{W}}^{2}\right]+\frac{\alpha}{2}\|z\|_{\mathcal{Z}}^{2} \\
& =\frac{1}{2} \sum_{k=1}^{Q} \omega_{k}\left\|\mathbf{C} u_{k}(z)-\bar{w}\right\|_{\mathcal{W}}^{2}+\frac{\alpha}{2}\|z\|_{\mathcal{Z}}^{2},
\end{aligned}
$$

where $u_{k}(z)$ is the solution of $(3.1)$ and $\omega_{k}=E\left[P_{k}\right]$ for $k=1, \ldots, Q$. This approach results in the semidiscretized optimization problem

$$
\min _{z \in \mathcal{Z}} J_{Q}(z), \quad \text { where } \quad u_{k}(z)=u_{k} \in \mathcal{V} \quad \text { solves }(3.1) \text { for } k=1, \ldots, Q .
$$

We note that for sparse grids, some weights $\omega_{k}$ are negative. Such cases require care. For example, if (2.2) is linear in $u$, then the original optimization problem (2.3) is convex quadratic in $z$. If some weights $\omega_{k}$ are negative, then it is not immediately obvious that the discretized problem (3.2) is convex or even well-posed. This issue is analyzed in [39] for linear state equations, and it is shown that for sufficiently fine collocation the discretized problem (3.2) is well-posed and the solution of (3.2) converges to the solution of original optimization problem (2.3) as the accuracy of the collocation method is increased. 
If for fixed $y \in \Gamma$ the mapping $v \mapsto \mathbf{N}(v, y)$ is Fréchet differentiable with Fréchet derivative $\mathbf{N}^{\prime}(v, y)$ (see Assumption 2.5), then the objective function in (3.3) is also Fréchet differentiable with gradient

$$
\nabla J_{Q}(z)=\alpha z+\sum_{k=1}^{Q} \omega_{k} \mathbf{B}_{k}^{*} p_{k},
$$

where $p_{k} \in \mathcal{V}$ solves the discrete adjoint equations

$$
\left(\mathbf{A}\left(y_{k}\right)+\mathbf{N}^{\prime}\left(u_{k}, y_{k}\right)\right)^{*} p_{k}+\mathbf{C}^{*}\left(\mathbf{C} u_{k}-\bar{w}\right)=0, \quad k=1, \ldots, Q .
$$

For the collocation discretization (3.2), the discrete adjoint equations (3.5) can be viewed as the collocation discretization of the adjoint equation (2.5), which is not necessarily the case for other stochastic collocation discretizations of the optimization problem; see [39].

3.3. Choice of collocation points: Generalized sparse grids. The objective function (2.3) requires integration, which potentially leads to many quadrature points in order to achieve desired levels of accuracy. This situation motivates our use of so-called sparse grids to compute the collocation points $\left\{y_{k}\right\}_{k=1}^{Q}$, the polynomials $\left\{P_{k}\right\}_{k=1}^{Q}$, and the corresponding weights $\left\{\omega_{k}\right\}_{k=1}^{Q}$. The material in this section is based on previous work by many authors, including [26, 28, 6, 3, 46, 48, 66].

The construction of Smolyak and tensor product rules begins with one-dimensional (1D) quadrature operators. For $k=1, \ldots, M$, let $\left\{E_{k}^{i}\right\}_{i \geq 1}$ denote a sequence of onedimensional quadrature operators such that

$$
E_{k}^{i}[f] \rightarrow E_{k}[f]=\int_{\Gamma_{k}} \rho_{k}(y) f(y) \mathrm{d} y \quad \text { as } \quad i \rightarrow \infty
$$

for $f \in C_{\rho_{k}}^{0}\left(\Gamma_{k}\right)$ sufficiently regular. We assume the quadrature operator $E_{k}^{i}$ is exact for polynomials of degree $d_{k}^{i}-1$, where $\left\{d_{k}^{i}\right\}_{i=1}^{\infty} \subset \mathbb{N}$ is an increasing sequence and $\mathcal{N}_{k}^{i}$ denotes the associated quadrature nodes. The tensor product quadrature rule for the integration of a function $f: \Gamma \rightarrow \mathbb{R}$ is $\left(E_{1}^{i} \otimes \cdots \otimes E_{M}^{i}\right) f$. The obvious disadvantage of this rule is that the number of quadrature nodes grows exponentially.

We define the $1 \mathrm{D}$ difference quadrature operators as

$$
\Delta_{k}^{1} \stackrel{\text { def }}{=} E_{k}^{1} \quad \text { and } \quad \Delta_{k}^{i} \stackrel{\text { def }}{=} E_{k}^{i}-E_{k}^{i-1} \quad \text { for } \quad i \geq 2 .
$$

Obviously, $E_{k}^{i}=\sum_{j=1}^{i} \Delta_{k}^{j}$; therefore, the tensor product quadrature rule can be written as

$$
\left(E_{1}^{i} \otimes \cdots \otimes E_{M}^{i}\right)[f]=\sum_{\max i_{k} \leq i}\left(\Delta_{1}^{i_{1}} \otimes \cdots \otimes \Delta_{M}^{i_{M}}\right)[f] .
$$

Since $E_{k}^{i}[f] \rightarrow E_{k}[f]=\int_{\Gamma_{k}} \rho_{k}(y) f(y) \mathrm{d} y$ as $i \rightarrow \infty$, we have

$$
\Delta_{k}^{i} f \rightarrow 0 \quad \text { as } \quad i \rightarrow \infty,
$$

where the rate of convergence depends on the smoothness of $f$. Therefore, we can approximate the right-hand side in (3.6) by

$$
\sum_{\sum_{k} i_{k} \leq i+M-1}\left(\Delta_{1}^{i_{1}} \otimes \cdots \otimes \Delta_{M}^{i_{M}}\right)[f] .
$$


Equation (3.7) is the Smolyak quadrature operator [58]. The approximation error of (3.7) is small provided the function $f$ is sufficiently smooth.

The Smolyak operator (3.7) can be generalized. Let $\mathbf{i}=\left(i_{1}, \ldots, i_{M}\right)$ be a multiindex and let $\mathcal{I} \subset \mathbb{N}_{+}^{M}$ be a multi-index set, where $\mathbb{N}_{+}=\{1,2, \ldots\}$. The generalized Smolyak quadrature operator is defined as

$$
E_{\mathcal{I}} \stackrel{\text { def }}{=} \sum_{\mathbf{i} \in \mathcal{I}} \Delta_{1}^{i_{1}} \otimes \cdots \otimes \Delta_{M}^{i_{M}} .
$$

For $\mathcal{I}=\left\{\left(i_{1}, \ldots, i_{M}\right): \max i_{k} \leq i\right\},(3.8)$ is the tensor product operator (3.6), and for $\mathcal{I}=\left\{\left(i_{1}, \ldots, i_{M}\right): \sum_{k} i_{k} \leq i+M-1\right\},(3.8)$ is the (classical) Smolyak quadrature operator (3.7).

We can write (3.8) in terms of the original 1D quadrature operators if the index set $\mathcal{I}$ is admissible.

Definition 3.1. The multi-index set $\mathcal{I} \subset \mathbb{N}_{+}^{M}$ is admissible if for all $\mathbf{i}=$ $\left(i_{1}, \ldots, i_{M}\right) \in \mathcal{I}$ the following holds:

$$
\mathbf{j}=\left(j_{1}, \ldots, j_{M}\right) \in \mathbb{N}_{+}^{M} \quad \text { and } \quad j_{k} \leq i_{k} \quad \forall k=1, \ldots, M \quad \Longrightarrow \quad \mathbf{j} \in \mathcal{I} .
$$

Let $|\mathbf{i}|_{1}=\sum_{k=1}^{M} i_{k}$, and define the characteristic function $\chi_{\mathcal{I}}(\mathbf{j})=1$ if $\mathbf{j} \in \mathcal{I}$ and zero otherwise. If $\mathcal{I}$ is admissible, then we can use the recombination technique [25] to write $(3.8)$ as

$$
E_{\mathcal{I}}=\sum_{\mathbf{i} \in \mathcal{I}}\left(\sum_{\mathbf{j} \in\{0,1\}^{M}}(-1)^{|\mathbf{j}|_{1}} \chi_{\mathcal{I}}(\mathbf{i}+\mathbf{j})\right)\left(E_{1}^{i_{1}} \otimes \cdots \otimes E_{M}^{i_{M}}\right) .
$$

Furthermore, by defining

$$
\vartheta(\mathbf{i}) \stackrel{\text { def }}{=} \sum_{\mathbf{j} \in\{0,1\}^{M}}(-1)^{|\mathbf{j}|_{1}} \chi_{\mathcal{I}}(\mathbf{i}+\mathbf{j}),
$$

we can determine the set of points required to evaluate $E_{\mathcal{I}}$ (i.e., the sparse grid associated with $\mathcal{I}$ ):

$$
\mathcal{N}_{\mathcal{I}} \stackrel{\text { def }}{=} \bigcup_{\{\mathbf{i} \in \mathcal{I}: \vartheta(\mathbf{i}) \neq 0\}}\left(\mathcal{N}_{1}^{i_{1}} \times \cdots \times \mathcal{N}_{M}^{i_{M}}\right)
$$

Similarly, the sparse-grid collocation weights can be computed from the weights of the original 1D quadrature formulas.

In general, the number of points in $\mathcal{N}_{\mathcal{I}}$ is considerably fewer than the number of points in the corresponding tensor product grid (unless $\mathcal{I}$ corresponds to the full tensor product rule). The number of points in $\mathcal{N}_{\mathcal{I}}$ can further be reduced if the $1 \mathrm{D}$ quadrature nodes are nested, that is, $\mathcal{N}_{k}^{i} \subset \mathcal{N}_{k}^{i+1}$ [48].

Convergence results for sparse-grid stochastic collocation methods for PDEs with random input data can be found, for example, in $[3,45,46,47]$. These papers generate sequences of multi-index sets in a predetermined manner. Our goal is to construct admissible index sets adaptively based on the progress of the optimization algorithm with the goal to keep the number of collocation points as small as possible while achieving a desired gradient tolerance for the optimization. One ingredient of our approach is the dimension-adaptive approach of [26], which we present next. 
3.4. Adaptive selection of index sets. We will determine the index set in (3.9) adaptively using the dimension-adaptive approach presented in [26].

If $\mathcal{I}$ is an admissible index set and $E_{\mathcal{I}}$ denotes the corresponding generalized sparse-grid quadrature operator, then the error associated with $E_{\mathcal{I}}$ applied to a function $f$ is

$$
E[f]-E_{\mathcal{I}}[f]=\sum_{\mathbf{j} \notin \mathcal{I}}\left(\Delta_{1}^{j_{1}} \otimes \cdots \otimes \Delta_{M}^{j_{M}}\right)[f] .
$$

Let $\mathcal{N}^{+}(\mathcal{I})=\{\mathbf{i} \notin \mathcal{I}: \mathcal{I} \cup\{\mathbf{i}\}$ is admissible $\}$ be the set of neighboring indices. The dimension adaptive approach of [26] approximates (3.10) by

$$
E[f]-E_{\mathcal{I}}[f] \approx \sum_{\mathbf{j} \in \mathcal{N}^{+}(\mathcal{I})}\left(\Delta_{1}^{j_{1}} \otimes \cdots \otimes \Delta_{M}^{j_{M}}\right)[f] .
$$

The algorithm proposed in [26] can be applied to vector-valued functions in $f \in$ $C_{\rho}^{0}(\Gamma ; \mathcal{Z})$ if we use a function $\wp: \mathcal{Z} \rightarrow[0, \infty)$ to quantify the size of the contribution $\left(\Delta_{1}^{j_{1}} \otimes \cdots \otimes \Delta_{M}^{j_{M}}\right)[f]$. (We will use the norm $\wp(\cdot)=\|\cdot\|_{\mathcal{Z}}$.) The dimension-adaptive sparse-grid algorithm [26] for functions in $C_{\rho}^{0}(\Gamma, \mathcal{Z})$ is adapted in Algorithm 3.2.

\section{Algorithm 3.2.}

Set $\mathbf{i}=(1, \ldots, 1), \mathcal{O}=\emptyset, \mathcal{A}=\{\mathbf{i}\}, r=\left(\Delta_{1}^{1} \otimes \cdots \otimes \Delta_{M}^{1}\right)[f], \eta=\eta_{\mathbf{i}}=\wp(r)$ while $\eta>$ TOL do

Select $\mathbf{i} \in \mathcal{A}$ corresponding to the largest $\eta_{\mathbf{i}}$

Set $\mathcal{A} \leftarrow \mathcal{A} \backslash\{\mathbf{i}\}$ and $\mathcal{O} \leftarrow \mathcal{O} \cup\{\mathbf{i}\}$

Update the error indicator $\eta \leftarrow \eta-\eta_{\mathbf{i}}$

for $k=1, \ldots, M$ do

Set $\mathbf{j}=\mathbf{i}+\mathbf{e}_{k}$

if $\mathcal{O} \cup \mathbf{j}$ is admissible then

Set $\mathcal{A} \leftarrow \mathcal{A} \cup\{\mathbf{j}\}$

Set $r_{\mathbf{j}}=\left(\Delta_{1}^{j_{1}} \otimes \cdots \otimes \Delta_{M}^{j_{M}}\right)[f]$

Set $\eta_{\mathbf{j}}=\wp\left(r_{\mathbf{j}}\right)$

Update the integral approximation $r \leftarrow r+r_{\mathbf{j}}$

Update the error indicator $\eta \leftarrow \eta+\eta_{\mathbf{j}}$

end if

end for

end while

4. Trust regions. The variation of the sparse grid allows us to use models of different fidelity in the optimization. We use the trust-region framework $[2,21]$ to adjust the model fidelity, in our case the sparse-grid collocation points, to the progress of the optimization algorithm. We first describe the trust-region framework, including the recent retrospective trust-region (RTR) algorithm [8], and then we describe how we compute our models when the optimization problem is given by (2.3), (3.2).

4.1. The algorithm. Trust-region algorithms for the solution of

$$
\min _{z \in \mathcal{Z}} J(z)
$$

Copyright $@$ by SIAM. Unauthorized reproduction of this article is prohibited. 
compute steps $z_{k+1}=z_{k}+s_{k} \in \mathcal{Z}$ that solve the trust-region subproblem

$$
\min _{s \in \mathcal{Z}} m_{k}(s) \quad \text { subject to } \quad\|s\|_{\mathcal{Z}} \leq \Delta_{k} .
$$

Here, $m_{k}: \mathcal{Z} \rightarrow \mathbb{R}$ is an approximation of $J$ around the point $z_{k}$, and $\Delta_{k}>0$ is the trust-region radius. In general, (4.1) need not be solved exactly. In fact, (4.1) need not have a solution since the set $\left\{s \in \mathcal{Z}:\|s\|_{\mathcal{Z}} \leq \Delta_{k}\right\}$ need not be compact for general Hilbert spaces $\mathcal{Z}$. The requirement on the accuracy of our solution to (4.1) is known as the fraction of Cauchy decrease condition

$$
m_{k}(0)-m_{k}\left(s_{k}\right) \geq \kappa_{0}\left\|\nabla m_{k}(0)\right\|_{\mathcal{Z}} \min \left\{\Delta_{k}, \frac{\left\|\nabla m_{k}(0)\right\|_{\mathcal{Z}}}{\beta_{k}}\right\}
$$

where $\kappa_{0} \in(0,1)$ and $\beta_{k}=1+\sup _{s \in \mathcal{B}_{k}}\left\|\nabla^{2} m_{k}(s)\right\|_{\mathcal{L}\left(\mathcal{Z}, \mathcal{Z}^{*}\right)}$. We make the following assumptions for the RTR method. Similar assumptions are made for the classical trust-region (CTR) method [21].

\section{Assumption 4.1 .}

1. $J$ is twice continuously Fréchet differentiable and bounded below.

2. $m_{k}$ is twice continuously Fréchet differentiable for $k=1,2, \ldots$.

3. There exist $\kappa_{1}>0, \kappa_{2}>1$ such that $\left\|\nabla^{2} J(z)\right\|_{\mathcal{L}\left(\mathcal{Z}, \mathcal{Z}^{*}\right)} \leq \kappa_{1}$ and $\left\|\nabla^{2} m_{k}(z)\right\|_{\mathcal{L}\left(\mathcal{Z}, \mathcal{Z}^{*}\right)} \leq \kappa_{2}-1$ for all $z \in \mathcal{Z}$ and for all $k=1,2, \ldots$

4. CTR algorithm: There exists $\xi>0$ such that

$$
\left\|\nabla m_{k}(0)-\nabla J\left(z_{k}\right)\right\|_{\mathcal{Z}} \leq \xi \min \left\{\left\|\nabla m_{k}(0)\right\|_{\mathcal{Z}}, \Delta_{k}\right\} .
$$

RTR algorithm: There exists $\xi>0$ such that

$$
\left\|\nabla m_{k}(0)-\nabla J\left(z_{k}\right)\right\|_{\mathcal{Z}} \leq \xi \min \left\{\left\|\nabla m_{k}(0)\right\|_{\mathcal{Z}}, \Delta_{k-1}\right\} .
$$

The inexact gradient condition (4.3) is adapted from [33].

Whether to accept $z_{k+1}=z_{k}+s_{k}$ as the new iterate or keep $z_{k+1}=z_{k}$ is decided based on the ratio between actual and predicted reduction,

$$
\varrho_{k}=\frac{J\left(z_{k}\right)-J\left(z_{k}+s_{k}\right)}{m_{k}(0)-m_{k}\left(s_{k}\right)} .
$$

In the CTR method, the new trust-region radius $\Delta_{k}$ is also determined based on $\varrho_{k}$. See, for example, [21]. In the RTR algorithm of [8] first a new model $m_{k+1}(s) \approx$ $J\left(\left(z_{k}+s_{k}\right)+s\right)$ is computed, and then the trust-region radius $\Delta_{k+1}$ is determined based on the ratio between actual and predicted reduction with the new model

$$
\tilde{\varrho}_{k+1}=\frac{J\left(z_{k}\right)-J\left(z_{k}+s_{k}\right)}{m_{k+1}\left(-s_{k}\right)-m_{k+1}(0)} .
$$

In our case the models $m_{k}$ are determined by (3.2) with a varying, small number of collocation points. A potential advantage of the RTR algorithm is that if the new model $m_{k+1}$ is better than $m_{k}$, the trust region may be increased faster, thereby allowing larger steps toward the solution. We will demonstrate both the classical and the RTR algorithms in our numerical examples. We list only the latter. 
AlgORIThM 4.2. RTR algorithm.

1. Initialization: Given $m_{k}, z_{k}, \Delta_{k}, 0<\gamma_{1} \leq \gamma_{2}<1, \Delta_{\max }>0,0<\eta_{0}<$ 1 , and $0<\eta_{1}<\eta_{2}<1$.

2. Step computation: Approximately solve the trust-region subproblem

$$
\min _{s \in \mathcal{Z}} m_{k}(s) \quad \text { subject to }\|s\|_{\mathcal{Z}} \leq \Delta_{k}
$$

for a step $s_{k}$ which satisfies (4.2).

3. Step acceptance: Compute $\varrho_{k}=\left(J\left(z_{k}\right)-J\left(z_{k}+s_{k}\right)\right) /\left(m_{k}(0)-m_{k}\left(s_{k}\right)\right)$.

if $\varrho_{k} \geq \eta_{0}$ then $z_{k+1}=z_{k}+s_{k} \quad$ else $\quad z_{k+1}=z_{k} \quad$ end if

4. Model update: Choose a new model, $m_{k+1}$, which satisfies (4.3), i.e.

$$
\left\|\nabla m_{k+1}(0)-\nabla J\left(z_{k+1}\right)\right\|_{\mathcal{Z}} \leq \xi \min \left\{\left\|\nabla m_{k+1}(0)\right\|_{\mathcal{Z}}, \Delta_{k}\right\} .
$$

\section{Trust-region update:}

if $z_{k+1}=z_{k}$ then $\quad \Delta_{k+1} \in\left(0, \gamma_{1}\left\|s_{k}\right\|_{\mathcal{Z}}\right]$

else Compute $\tilde{\varrho}_{k+1}=\left(J\left(z_{k}\right)-J\left(z_{k+1}\right)\right) /\left(m_{k+1}\left(-s_{k}\right)-m_{k+1}(0)\right)$ and update $\Delta_{k+1}$ by

$\begin{array}{llll}\text { if } \tilde{\varrho}_{k+1} \leq \eta_{1} & \text { then } & \Delta_{k+1} \in\left(0, \gamma_{2}\left\|s_{k}\right\|_{\mathcal{Z}}\right] & \text { end if } \\ \text { if } \tilde{\rho}_{k+1} \in\left(\eta_{1}, \eta_{2}\right) & \text { then } & \Delta_{k+1} \in\left[\gamma_{2}\left\|s_{k}\right\|_{\mathcal{Z}}, \Delta_{k}\right] & \text { end if } \\ \text { if } \tilde{\varrho}_{k+1} \geq \eta_{2} & \text { then } & \Delta_{k+1} \in\left[\Delta_{k}, \Delta_{\text {max }}\right] & \text { end if }\end{array}$

In the CTR algorithm, $\Delta_{k}$ is updated based on $\varrho_{k}$ instead of $\tilde{\varrho}_{k+1}$, and the new model is computed after the trust-region update.

Under Assumption 4.1, one can prove the first-order convergence of Algorithm 4.2. This is a slight generalization over the convergence result in [8], where $\nabla m_{k}(0)=$ $\nabla J\left(z_{k}\right)$ is assumed instead of (4.3).

TheOREm 4.3. Suppose Assumption 4.1 holds. Then the iterates $\left\{z_{k}\right\}$ generated by Algorithm 4.2 satisfy

$$
\liminf _{k \rightarrow \infty}\left\|\nabla m_{k}(0)\right\|_{\mathcal{Z}}=\liminf _{k \rightarrow \infty}\left\|\nabla J\left(z_{k}\right)\right\|_{\mathcal{Z}}=0 .
$$

The proof is given in the appendix. The same result holds for the CTR algorithm.

We stop the trust-region method if either $\left\|s_{k}\right\|_{\mathcal{Z}} \leq$ stol for a step tolerance stol or $\left\|\nabla m_{k}(0)\right\|_{\mathcal{Z}}<$ gtol for a gradient tolerance gtol. The inexact gradient condition (4.3) implies $\left\|\nabla J\left(z_{k}\right)\right\|_{\mathcal{Z}} \leq(1+\xi)\left\|\nabla m_{k}(0)\right\|_{\mathcal{Z}} \leq(1+\xi)$ gtol.

4.2. The gradient condition and adaptive sparse grids. To solve the optimization problem (2.3), we define the models in Algorithm 4.2 as the stochastic collocation approximations

$$
m_{k}(s) \stackrel{\text { def }}{=} J_{Q_{k}}\left(z_{k}+s\right)=\frac{1}{2} E_{\mathcal{I}_{k}}\left[\left\|\mathbf{C} u\left(z_{k}+s\right)-\bar{w}\right\|_{\mathcal{W}}^{2}\right]+\frac{\alpha}{2}\left\|z_{k}+s\right\|_{\mathcal{Z}}^{2},
$$

where $z_{k} \in \mathcal{Z}, \mathcal{I}_{k} \subset \mathbb{N}_{+}^{M}$ is an admissible index set, $\mathcal{N}_{\mathcal{I}_{k}}$ is the set of sparse-grid collocation points, and $Q_{k}=\left|\mathcal{N}_{\mathcal{I}_{k}}\right|$. The specific forms of $\nabla J(z)$ and $\nabla J_{\mathcal{I}_{k}}(z)$ give rise to the gradient error

$$
\left\|\nabla J(z)-\nabla J_{\mathcal{I}_{k}}(z)\right\|_{\mathcal{Z}}=\left\|\sum_{\mathbf{i} \notin \mathcal{I}_{k}}\left(\Delta_{1}^{i_{1}} \otimes \cdots \otimes \Delta_{M}^{i_{M}}\right)\left[\mathbf{B}^{*} p\right]\right\|_{\mathcal{Z}}
$$

Copyright (c) by SIAM. Unauthorized reproduction of this article is prohibited. 
and the inexact gradient condition, (4.3),

$$
\begin{aligned}
& \left\|\sum_{\mathbf{i} \notin \mathcal{I}_{k}}\left(\Delta_{1}^{i_{1}} \otimes \cdots \otimes \Delta_{M}^{i_{M}}\right)\left[\mathbf{B}^{*} p\right]\right\|_{\mathcal{Z}} \\
& \quad \leq \xi \min \left\{\left\|\alpha z+\sum_{\mathbf{i} \in \mathcal{I}_{k}}\left(\Delta_{1}^{i_{1}} \otimes \cdots \otimes \Delta_{M}^{i_{M}}\right)\left[\mathbf{B}^{*} p\right]\right\|_{\mathcal{Z}}, \Delta_{k-1}\right\} .
\end{aligned}
$$

We now define the set $\mathcal{A}_{M} \stackrel{\text { def }}{=}\left\{\mathcal{I} \subset \mathbb{N}^{M}: \mathcal{I}\right.$ is admissible $\}$. To keep the number of collocation points small and still satisfy (4.3), we ideally want to compute the index set $\mathcal{I}_{k}$ as a solution of

$$
\min _{\mathcal{I} \subset \mathcal{A}_{M}}\left|\mathcal{N}_{\mathcal{I}}\right| \text { subject to }\left\|\nabla J\left(z_{k}\right)-\nabla J_{\mathcal{I}}\left(z_{k}\right)\right\|_{\mathcal{Z}} \leq \xi \min \left\{\left\|\nabla J_{\mathcal{I}}\left(z_{k}\right)\right\|_{\mathcal{Z}}, \Delta_{k-1}\right\},
$$

where $\left|\mathcal{N}_{\mathcal{I}}\right|$ denotes the number of collocation points associated with $E_{\mathcal{I}}$. The problem (4.5) is combinatorial and expensive to solve. Instead of solving (4.4) exactly, we employ Algorithm 3.2 with $\wp(\cdot)=\|\cdot\|_{\mathcal{Z}}$. We use the the global error indicator

$$
\eta \stackrel{\text { def }}{=} \sum_{\mathbf{i} \in \mathcal{N}^{+}(\mathcal{I})}\left\|\left(\Delta_{1}^{i_{1}} \otimes \cdots \otimes \Delta_{M}^{i_{M}}\right)\left[\mathbf{B}^{*} p\right]\right\|_{\mathcal{Z}} \approx \sum_{\mathbf{i} \notin \mathcal{I}}\left\|\left(\Delta_{1}^{i_{1}} \otimes \cdots \otimes \Delta_{M}^{i_{M}}\right)\left[\mathbf{B}^{*} p\right]\right\|_{\mathcal{Z}} .
$$

Note that if there exists $C>0$ such that $\sum_{\mathbf{i} \notin \mathcal{I}}\left\|\left(\Delta_{1}^{i_{1}} \otimes \cdots \otimes \Delta_{M}^{i_{M}}\right)\left[\mathbf{B}^{*} p\right]\right\|_{\mathcal{Z}} \leq C \eta$, then (4.3) is satisfied, and global convergence is ensured. Such a result is problemdependent, however, and is not known for the examples in section 5.

The new model $m_{k+1}$ for our problem (2.3), (3.2) is chosen as follows.

AlgORITHM 4.4. Adaptive stochastic collocation model selection.

Given $\mathcal{I}_{k} \subset \mathbb{N}_{+}^{M}$. Set $\mathcal{A}=\mathcal{N}^{+}\left(\mathcal{I}_{k}\right), \mathcal{O}=\mathcal{I}_{k}, r_{\mathbf{i}}=\left(\Delta_{1}^{i_{1}} \otimes \cdots \otimes \Delta_{M}^{i_{M}}\right)\left[\mathbf{B}^{*} p\right]$ and $\eta_{\mathbf{i}}=\left\|r_{\mathbf{i}}\right\|_{\mathcal{Z}}$ for $\mathbf{i} \in \mathcal{A} \cup \mathcal{O}, \eta=\sum_{\mathbf{i} \in \mathcal{A}} \eta_{\mathbf{i}}, g=\alpha z+\sum_{\mathbf{i} \in \mathcal{O}} r_{\mathbf{i}}$, and TOL $=$ $\xi \min \left\{\|g\|_{\mathcal{Z}}, \Delta_{k-1}\right\}$

while $\eta>$ TOL do

Select $\mathbf{i} \in \mathcal{A}$ corresponding to the largest $\eta_{\mathbf{i}}$

Set $\mathcal{A} \leftarrow \mathcal{A} \backslash\{\mathbf{i}\}$ and $\mathcal{O} \leftarrow \mathcal{O} \cup\{\mathbf{i}\}$

Update the error indicator $\eta \leftarrow \eta-\eta_{\mathbf{i}}$

for $\mathrm{k}=1, \ldots, \mathrm{M}$ do

Set $\mathbf{j}=\mathbf{i}+\mathbf{e}_{k}$

if $\mathcal{O} \cup\{\mathbf{j}\}$ is admissible then

Set $\mathcal{A} \leftarrow \mathcal{A} \cup\{\mathbf{j}\}$

Set $r_{\mathbf{j}}=\left(\Delta_{1}^{j_{1}} \otimes \cdots \otimes \Delta_{M}^{j_{M}}\right)\left[\mathbf{B}^{*} p\right]$

Set $\eta_{\mathbf{j}}=\left\|r_{\mathbf{j}}\right\|_{\mathcal{Z}}$

Update the gradient approximation $g \leftarrow g+r_{\mathbf{j}}$

Update the error indicator $\eta \leftarrow \eta+\eta_{\mathbf{i}}$

Updated the stopping tolerance TOL $=\xi \min \left\{\|g\|_{\mathcal{Z}}, \Delta_{k-1}\right\}$

end if

end for

end while

Set $\mathcal{I}_{k+1}=\mathcal{A} \cup \mathcal{O}$ and $m_{k+1}(s)=J_{\mathcal{I}_{k+1}}\left(z_{k}+s\right)$.

If the CTR method is used, the tolerance in Algorithm 4.4 is set to TOL $=$ $\xi \min \left\{\|g\|_{\mathcal{Z}}, \Delta_{k}\right\} ;$ see $(4.3)$.

Copyright $@$ by SIAM. Unauthorized reproduction of this article is prohibited. 
5. Numerical results. In the following sections, we present the results of applying our adaptive framework to three numerical examples: optimal control of the $1 \mathrm{D}$ diffusion equation with a discontinuous diffusion coefficient, optimal control of the steady 1D Burgers equation, and optimal control of the two-dimensional (2D) Helmholtz equation. First we give a summary of the computational tools.

Algorithms. For the discontinuous diffusion and Burgers examples we employ the CTR algorithm [21] and the RTR algorithm [8] to guide adaptivity. We compare the results of CTR and RTR with those of a Newton-CG algorithm applied to a fixed highfidelity stochastic collocation discretization of the optimization problem [38]. For the Helmholtz example, only the CTR algorithm is used, and no comparisons are made to the high-fidelity Newton-CG algorithm because of its prohibitive computational cost. In all examples, the stochastic models are computed by using Algorithm 4.4. We use the Steihaug-Toint CG method to approximate the solution of the trust-region subproblem (4.1).

Implementations. The discontinuous diffusion example in section 5.1 involves a small spatial discretization and two random fields and is implemented in MATLAB. In contrast, in the Helmholtz example in section 5.3, the size of the spatial discretization and the large number of stochastic variables (up to 40) mandate a large-scale computational infrastructure and an efficient software implementation. The Burgers example in section 5.2, with a smaller spatial discretization and four random fields, uses the same software base. Our software is built on a variety of Trilinos [34, 35] packages. A crucial component is the discretization library Intrepid [10], which is responsible for both the spatial finite element and the adaptive stochastic discretizations. Our optimization algorithms are implemented outside of Trilinos; in order to achieve full generality, the implementations are based on abstractions of vector spaces, through inheritance-based polymorphism. In other words, they are problem-independent. Computations of problem-specific quantities, such as objective function values, gradients, and Hessian-vector products, are implemented by using two layers of parallelism, through MPI groups. One layer of parallelism is responsible for the stochastic collocation and requires minimal communication; the other, communication-intensive layer handles distributed linear algebra and enables, for instance, an efficient parallel multigrid solution of the forward and adjoint PDEs, whenever applicable. ${ }^{1}$

Computational infrastructure. The numerical experiments for the Helmholtz example in section 5.3 are carried out on RedSky, an institutional computing cluster at Sandia National Laboratories. The cluster is built on a three-dimensional toroidal QDR InfiniBand interconnect and provides 2,816 compute nodes, each with 8 cores, $2.93 \mathrm{GHz}$ Nehalem X5570 processors, and 12 GB RAM per compute node.

5.1. Optimal control of an elliptic equation with discontinuous coefficient. The governing equation in our first example is a $1 \mathrm{D}$ elliptic equation with a discontinuous diffusion coefficient. We assume that the interface of the discontinuity is uncertain but that the values of the piecewise constant coefficient on the two subdomains are known. This problem is motivated by, for example, subsurface flow control through fractured media, in which the locations of the fractures are uncertain.

\footnotetext{
${ }^{1}$ Since the multigrid solvers currently offered by the Trilinos package ML are unsuitable for the Helmholtz equation and unnecessary for the 1D Burgers example, we use the sparse direct solver KLU from the Trilinos package Amesos. This limits the exercised parallelism to the stochastic collocation layer. We are documenting the simultaneous use of distributed stochastic collocation and Trilinos/ML multigrid solvers in a related publication, in the context of the optimal control of three-dimensional Navier-Stokes equations.
} 
In this example, the spatial domain is $D=(-1,1)$ and the stochastic image space is $\Gamma=\Gamma_{1} \times \Gamma_{2}=[-0.1,0.1] \times[-0.5,0.5]$, endowed with the uniform probability density. Furthermore, the problem coefficients are defined as

$$
\epsilon(y, x)=\epsilon_{l} \chi_{\left(-1, y_{1}\right)}(x)+\epsilon_{r} \chi_{\left(y_{1}, 1\right)}(x) \quad \text { with } \quad \epsilon_{l}=0.1, \epsilon_{r}=10,
$$

and $f(y, x)=\exp \left(-\left(x-y_{2}\right)^{2}\right)$. We consider the optimal control problem

$$
\min _{z \in L^{2}(D)} \frac{1}{2} \int_{\Gamma} \rho(y) \int_{-1}^{1}(u(y, x ; z)-1)^{2} \mathrm{~d} x \mathrm{~d} y+\frac{\alpha}{2} \int_{-1}^{1} z(x)^{2} \mathrm{~d} x,
$$

where $\alpha=10^{-4}$ and $u(y ; \cdot ; z) \in H_{0}^{1}(D)$ solves

$$
\begin{array}{crr}
-\partial_{x}\left(\epsilon(y, x) \partial_{x} u(y, x)\right)=f(y, x)+z(x), & y \in \Gamma, & x \in(-1,1), \\
u(y,-1)=0, \quad u(y, 1)=0, & y \in \Gamma .
\end{array}
$$

5.1.1. The infinite-dimensional problem. To verify the assumptions in section 2, we first focus on the state equation (5.2). The numerical solution of a 2D elliptic interface problem with fixed interface and random diffusivities on each subdomain is considered in [71]. In our case the interface is random; therefore, the analysis is more involved. form

Using the Lax-Milgram lemma, we can show that given $z \in L^{2}(D)$, the weak

$$
\int_{\Gamma} \rho(y) \int_{-1}^{1} \epsilon(y, x) \partial_{x} u(y, x) \partial_{x} \psi(y, x)-(f(y, x)+z(x)) \psi(y, x) d x d y=0
$$

for all $\psi \in L_{\rho}^{2}\left(\Gamma, H_{0}^{1}(D)\right)$ has a unique solution $u(\cdot ; z) \in L_{\rho}^{2}\left(\Gamma, H_{0}^{1}(D)\right)$. Moreover, for every $y \in \Gamma$

$$
\int_{-1}^{1} \epsilon(y, x) \partial_{x} u(y, x) \partial_{x} \psi(y, x)-(f(y, x)+z(x)) \psi(x) d x=0 \quad \forall \psi \in H_{0}^{1}(D)
$$

has a unique solution $u(y ; z) \in H_{0}^{1}(D)$, and there exists a constant $c>0$ independent of $y \in \Gamma$ and $z$ such that $\|u(y ; z)\|_{H_{0}^{1}(D)} \leq c\left(1+\|z\|_{L^{2}(D)}\right)$. However, analyzing the smoothness properties of this solution with respect to $y$ (more precisely, with respect to the interface condition $y_{1}$ ) is more involved.

The sensitivity of the solution $u(y, \cdot ; z)$ with respect to the interface location $y_{1}$ is a function $s(y, x)$ that is, in general, no longer continuous at the interface $x=y_{1}$; see [15]. Therefore the sensitivity $s$ of the solution $u(y, \cdot ; z)$ is not an $H_{0}^{1}(D)$ function, and hence the standard setting above cannot be used.

To analyze the dependence of the solution of (5.2) on $y_{1}$, we use a domain decomposition formulation (see, e.g., [54]). We note that the solution of (5.2) satisfies the interface condition

$$
\epsilon_{l} \partial_{x} u(y, x)=\epsilon_{r} \partial_{x} u(y, x) \quad \text { at } x=y_{1} .
$$

Let $v_{l} \in H^{1}\left(-1, y_{1}\right), v_{r} \in H^{1}\left(y_{1}, 1\right)$ be functions with $v_{l}\left(y_{1}\right)=1=v_{r}\left(y_{1}\right)$ and $v_{l}(-1)=0=v_{r}(1)$. We write

$$
u(y, x)= \begin{cases}u_{l}(y, x)+d v_{l}(x), & x \in\left[-1, y_{1}\right], \\ u_{r}(y, x)+d v_{r}(x), & x \in\left[y_{1}, 1\right],\end{cases}
$$

Copyright $@$ by SIAM. Unauthorized reproduction of this article is prohibited. 
with $u_{l}(y, \cdot) \in H_{0}^{1}\left(-1, y_{1}\right), u_{r}(y, \cdot) \in H_{0}^{1}\left(y_{1}, 1\right)$, and $d \in \mathbb{R}$. We insert the representation into (5.2), multiply by the test functions $\phi_{l} \in H_{0}^{1}\left(-1, y_{1}\right), \phi_{r} \in H_{0}^{1}\left(y_{1}, 1\right)$ (extended by zero onto $(-1,1)$ ), and integrate by parts. Next, we perform the variable transformations

$$
x=\left\{\begin{array}{cl}
-1+\left(y_{1}+1\right) \xi \in\left[-1, y_{1}\right] & \text { for } \xi \in[0,1], \\
1+\left(y_{1}-1\right) \xi \in\left[y_{1}, 1\right] & \text { for } \xi \in[0,1],
\end{array}\right.
$$

and we define

$$
\begin{aligned}
\widehat{u}_{l}(\xi) & =u_{l}\left(-1+\left(y_{1}+1\right) \xi\right), & \widehat{u}_{r}(\xi) & =u_{r}\left(1+\left(y_{1}-1\right) \xi\right), \\
\widehat{v}_{l}(\xi) & =v_{l}\left(-1+\left(y_{1}+1\right) \xi\right), & \widehat{v}_{r}(\xi) & =v_{r}\left(1+\left(y_{1}-1\right) \xi\right), \\
\widehat{z}_{l}(\xi) & =z\left(-1+\left(y_{1}+1\right) \xi\right), & \widehat{z}_{r}(\xi) & =z\left(1+\left(y_{1}-1\right) \xi\right), \\
\widehat{f}_{l}\left(y_{2}, \xi\right) & =f\left(y_{2},-1+\left(y_{1}+1\right) \xi\right), & \widehat{f}_{r}\left(y_{2}, \xi\right) & =f\left(y_{2}, 1+\left(y_{1}-1\right) \xi\right) .
\end{aligned}
$$

The weak form of (5.2) is equivalent to the system

$$
\begin{aligned}
& \left(1+y_{1}\right)^{-1} \int_{0}^{1} \epsilon_{l} \partial_{\xi}\left(\widehat{u}_{l}+d \widehat{v}_{l}\right) \partial_{\xi} \phi \mathrm{d} \xi=\left(1+y_{1}\right) \int_{0}^{1}\left(\widehat{f}_{l}\left(y_{2}, \cdot\right)+\widehat{z}_{l}\right) \phi \mathrm{d} \xi \\
& \left(1-y_{1}\right)^{-1} \int_{0}^{1} \epsilon_{r} \partial_{\xi}\left(\widehat{u}_{r}+d \widehat{v}_{r}\right) \partial_{\xi} \psi \mathrm{d} \xi=\left(1-y_{1}\right) \int_{0}^{1}\left(\widehat{f}_{r}\left(y_{2}, \cdot\right)+\widehat{z}_{r}\right) \psi \mathrm{d} \xi \\
& \left(1+y_{1}\right)^{-1} \int_{0}^{1} \epsilon_{l} \partial_{\xi}\left(\widehat{u}_{l}+d \widehat{v}_{l}\right) \partial_{\xi} \widehat{v}_{l} \mathrm{~d} \xi+\left(1-y_{1}\right)^{-1} \int_{0}^{1} \epsilon_{r} \partial_{\xi}\left(\widehat{u}_{r}+d \widehat{v}_{r}\right) \partial_{\xi} \widehat{v}_{r} \mathrm{~d} \xi \\
& =\left(1+y_{1}\right) \int_{0}^{1}\left(\widehat{f}_{l}\left(y_{2}, \cdot\right)+\widehat{z}_{l}\right) \widehat{v}_{l} \mathrm{~d} \xi+\left(1-y_{1}\right) \int_{0}^{1}\left(\widehat{f}_{r}\left(y_{2}, \cdot\right)+\widehat{z}_{r}\right) \partial_{\xi} \widehat{v}_{r} \mathrm{~d} \xi
\end{aligned}
$$

for all $\phi, \psi \in H_{0}^{1}(0,1)$. We have omitted the argument $(\xi)$ in $(5.5 \mathrm{a})-(5.5 \mathrm{c})$. Equation $(5.5 \mathrm{c})$ is just a weak form of the interface condition (5.4). The left-hand side in (5.5) defines a bounded linear operator

$$
\mathbf{A}\left(y_{1}\right)=\left(\begin{array}{ccc}
A_{l l}\left(y_{1}\right) & 0 & A_{l d}\left(y_{1}\right) \\
0 & A_{r r}\left(y_{1}\right) & A_{r d}\left(y_{1}\right) \\
A_{d l}\left(y_{1}\right) & A_{d r}\left(y_{1}\right) & A_{d d}\left(y_{1}\right)
\end{array}\right)
$$

from $H_{0}^{1}(0,1) \times H_{0}^{1}(0,1) \times \mathbb{R}$ to $H^{-1}(0,1) \times H^{-1}(0,1) \times \mathbb{R}$. For example, $A_{l l}(y)$ and $A_{l d}(y)$ are defined by

$$
\begin{aligned}
\left\langle A_{l l}\left(y_{1}\right) \widehat{u}_{l}, \phi\right\rangle_{H^{-1} \times H_{0}^{1}} & =\left(1+y_{1}\right)^{-1} \int_{0}^{1} \epsilon_{l} \partial_{\xi} \widehat{u}_{l}(\xi) \partial_{\xi} \phi(\xi) \mathrm{d} \xi \quad \text { and } \\
\left\langle A_{l d}\left(y_{1}\right) d, \phi\right\rangle_{H^{-1} \times H_{0}^{1}} & =\left(1+y_{1}\right)^{-1} \int_{0}^{1} \epsilon_{l} d \partial_{\xi} \widehat{v}_{l}(\xi) \partial_{\xi} \phi(\xi) \mathrm{d} \xi
\end{aligned}
$$

respectively. The other block operators are defined analogously. The right-hand side in (5.5) leads to the operator

$$
\mathbf{B}\left(y_{1}\right)=\left(\begin{array}{cc}
B_{l l}\left(y_{1}\right) & 0 \\
0 & B_{r r}\left(y_{1}\right) \\
B_{d l}\left(y_{1}\right) & B_{d l}\left(y_{1}\right)
\end{array}\right)
$$

Copyright (c) by SIAM. Unauthorized reproduction of this article is prohibited. 
from $L^{2}(0,1) \times L^{2}(0,1)$ to $H^{-1}(0,1) \times H^{-1}(0,1) \times \mathbb{R}$, and the functional

$$
-\mathbf{b}\left(y_{1}, y_{2}\right)=\left(\begin{array}{c}
l_{l}\left(y_{1}, y_{2}\right) \\
l_{r}\left(y_{1}, y_{2}\right) \\
l_{d}\left(y_{1}, y_{2}\right)
\end{array}\right) \in H^{-1}(0,1) \times H^{-1}(0,1) \times \mathbb{R} .
$$

For example,

$$
\begin{aligned}
\left\langle B_{l l}\left(y_{1}\right) \widehat{z}_{l}, \phi\right\rangle_{H^{-1} \times H_{0}^{1}} & =-\left(1+y_{1}\right) \int_{0}^{1} \widehat{z}_{l}(\xi) \phi(\xi) \mathrm{d} \xi \text { and } \\
B_{d l}\left(y_{1}\right) \widehat{z}_{l} & =-\left(1+y_{1}\right) \int_{0}^{1} \widehat{z}_{l}(\xi) \widehat{v}_{l}(\xi) \mathrm{d} \xi \in \mathbb{R} .
\end{aligned}
$$

The system (5.5) can now be written as

$$
\mathbf{A}\left(y_{1}\right)\left(\begin{array}{c}
\widehat{u}_{l} \\
\widehat{u}_{r} \\
d
\end{array}\right)+\mathbf{B}\left(y_{1}\right)\left(\begin{array}{c}
\widehat{z}_{l} \\
\widehat{z}_{r}
\end{array}\right)+\mathbf{b}\left(y_{1}, y_{2}\right)=0 .
$$

We set $\mathcal{V}=H_{0}^{1}(0,1) \times H_{0}^{1}(0,1) \times \mathbb{R}$ and $\mathcal{Z}=L^{2}(0,1) \times L^{2}(0,1)$

Using the relation between (5.3) and (5.9), one can show that for any $y_{1} \in \Gamma_{1}$ the linear operator (5.6) has a bounded inverse; that is, one can show the existence of a constant $c>0$ independent of $y_{1} \in \Gamma_{1}$ such that

$$
\left\|\mathbf{A}\left(y_{1}\right)^{-1}\right\|_{\mathcal{L}\left(\mathcal{V}^{*}, \mathcal{V}\right)} \leq c \quad \forall y_{1} \in \Gamma_{1} .
$$

See, for example, [54]. Therefore, for any $y=\left(y_{1}, y_{2}\right) \in \Gamma, \widehat{z}_{l} \in L^{2}(0,1), \widehat{z}_{2} \in L^{2}(0,1)$ the system (5.9) has a unique solution $\left(\widehat{u}_{l}(z ; y, \cdot), \widehat{u}_{r}(z ; y, \cdot), d(z ; y)\right) \in H_{0}^{1}(0,1) \times$ $H_{0}^{1}(0,1) \times \mathbb{R}$.

From the definitions (5.6), (5.7), and (5.8) it follows immediately that $y_{1} \mapsto \mathbf{A}\left(y_{1}\right)$, $y_{1} \mapsto \mathbf{B}\left(y_{1}\right),\left(y_{1}, y_{2}\right) \mapsto \mathbf{b}\left(y_{1}, y_{2}\right)$ are Fréchet differentiable. Therefore, the implicit function theorem applied to (5.5) implies the Fréchet differentiability of $\Gamma \ni y \mapsto$ $\left(\widehat{u}_{l}(z ; y, \cdot), \widehat{u}_{r}(z ; y, \cdot), d(z ; y)\right) \in H_{0}^{1}(0,1) \times H_{0}^{1}(0,1) \times \mathbb{R}$.

We have shown that Assumption 2.2 is satisfied for (5.9). Since the state equation (5.9) is linear, the assumptions of Theorem 2.4 are satisfied, as is Assumption 2.5.

5.1.2. Discretization and numerical results. We discretize the state equation (5.5) using continuous piecewise linear finite elements. The finite-element mesh used for the state variables is uniform on $\left[-1, y_{1}\right]$ with $N_{l}=64$ intervals and is uniform on $\left[y_{1}, 1\right]$ with $N_{r}=64$ intervals. The control variable is discretized on a uniform mesh on $[-1,1]$ with $N=128$ intervals. The resulting discretized system is identical to the system obtained by applying continuous piecewise linear finite elements directly to the weak form of (5.2), and this is how we implement it.

For a high-fidelity discretization in $\Gamma$, we use a level 7 isotropic Smolyak sparse grid built on 1D Gauss-Patterson knots $(Q=1793)$.

The optimization results are depicted in Figure 5.1. The bottom images depict the adapted sparse grid index set and corresponding sparse grid at the final optimization iteration. The index set displays anisotropic behavior with regard to the random coefficients. In fact, considerable effort is required to resolve the discontinuous diffusion coefficients $\left(y_{1}\right)$. Exploiting this anisotropy results in a significantly smaller adapted sparse grid $(Q=417)$ and, hence, far fewer PDE solves. The top row depicts the 

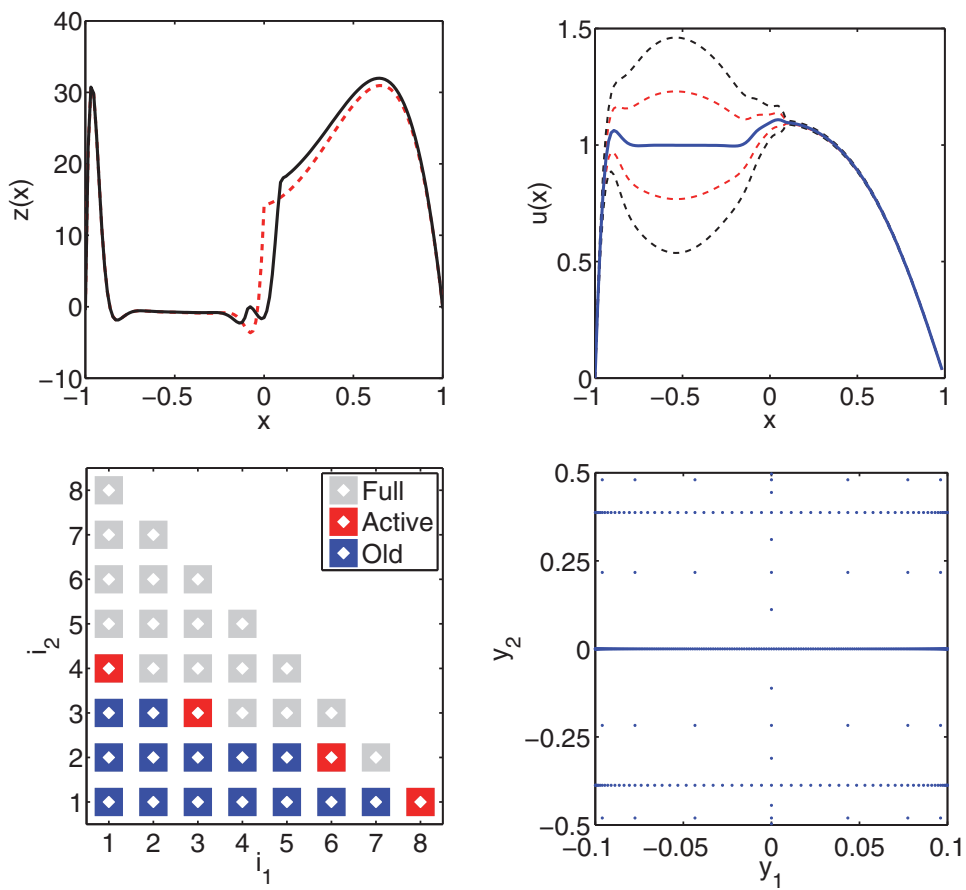

FIG. 5.1. Top left: Computed optimal control and mean value control (i.e., y replaced by $E[y]=0$, red dashed line). Top right: Expected value of computed optimal state with one and two standard deviation intervals added. Bottom left: Generalized sparse grid index set. The red blocks denote "active" indices and the blue blocks denote "old" indices. The gray blocks denote the indices in the isotropic Smolyak index set of level eight. Bottom right: Collocation points corresponding to the index set $\mathcal{I}=\mathcal{A} \cup \mathcal{O}$.

optimal controls for the stochastic problem and the deterministic substitute problem in which $y$ is replaced by $E[y]=0$ (red dashed line). Also depicted is the expected value of the state plus one and two standard deviation intervals.

The iteration history for the RTR algorithm is listed in Table 5.1, and an algorithm comparison is listed in Table 5.2. Table 5.1 highlights the fact that most work in the trust-region algorithm is performed on small sparse grids $(Q \leq 65)$, which results in about a five- to sixfold reduction in the number of PDE solves when compared

TABLE 5.1

Iteration history of the RTR algorithm applied to Example 5.1. Here $k$ is the number of trustregion iterations, $\widehat{J}\left(z_{k}\right)$ is the objective function value, $\left\|\nabla \widehat{J_{\mathcal{I}}}\left(z_{k}\right)\right\|_{\mathcal{Z}}$ is the model gradient norm value, $\left\|s_{k}\right\|_{\mathcal{Z}}$ is the step size, $\Delta_{k}$ is the trust-region radius, $C G$ is the number of Steihaug-Toint $C G$ iterations, Adaptive is the number of sparse grid adaptation iterations, and $Q$ is the number of collocation points.

\begin{tabular}{cccrcccc}
\hline$k$ & $\widehat{J}\left(z_{k}\right)$ & $\left\|\nabla \widehat{J}_{\mathcal{I}}\left(z_{k}\right)\right\|_{\mathcal{Z}}$ & $\left\|s_{k}\right\|_{\mathcal{Z}}$ & $\Delta_{k}$ & $\mathrm{CG}$ & Adaptive & $Q$ \\
\hline 0 & $5.660714 \mathrm{e}-01$ & $2.559392 \mathrm{e}-01$ & - & 1000 & - & - & 1 \\
1 & $2.183827 \mathrm{e}-01$ & $1.789019 \mathrm{e}-02$ & 153.89019 & 2500 & 5 & 2 & 9 \\
2 & $1.455063 \mathrm{e}-01$ & $9.838275 \mathrm{e}-04$ & 47.32745 & 5000 & 4 & 1 & 17 \\
3 & $1.265783 \mathrm{e}-01$ & $4.369266 \mathrm{e}-05$ & 90.04826 & 5000 & 10 & 2 & 33 \\
4 & $1.263875 \mathrm{e}-01$ & $2.692335 \mathrm{e}-06$ & 11.51365 & 5000 & 11 & 3 & 65 \\
5 & $1.263851 \mathrm{e}-01$ & $1.906452 \mathrm{e}-07$ & 2.77416 & 5000 & 16 & 4 & 161 \\
6 & $1.263851 \mathrm{e}-01$ & $2.410386 \mathrm{e}-08$ & 0.18155 & 5000 & 15 & 2 & 417 \\
\hline
\end{tabular}

Copyright (c) by SIAM. Unauthorized reproduction of this article is prohibited. 
TABLE 5.2

Total number of outer iterations (Iter), adaptive steps (Adaptive), and PDE solves (PDE), and final number of collocation points used for the model $(Q)$ for the three algorithms applied to Example 5.1. The fourth column contains the ratio of the total number of PDE solves for Newton-CG versus the total number of PDE solves for the other algorithms (Ratio).

\begin{tabular}{lccrrc}
\hline Algorithm & Iter & Adaptive & PDE & $Q$ & Ratio \\
\hline Newton-CG & 3 & 0 & 218,746 & 1,793 & 1.00 \\
CTR & 6 & 14 & 35,906 & 417 & 6.09 \\
RTR & 6 & 14 & 46,664 & 417 & 4.69 \\
\hline
\end{tabular}

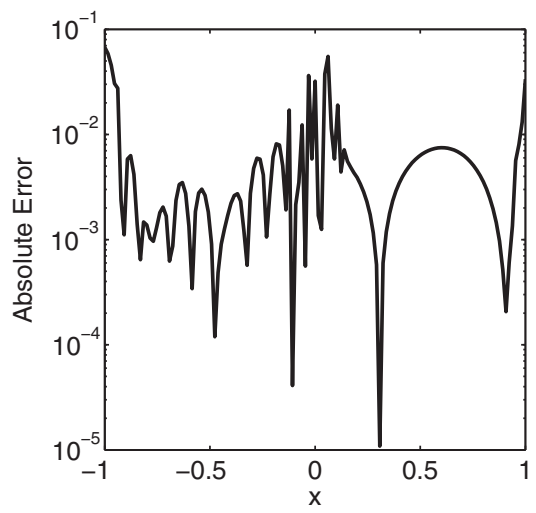

FIG. 5.2. Absolute error of the control computed using adaptive sparse grids and the control computed using a tensor product grid.

with the Newton-CG. In this case, all iterations for the RTR as well as for the CTR algorithm are successful, and both algorithms generate the same trust-region radii $\Delta_{k}$. The RTR algorithm requires the evaluation of the new model at the previous iterate, $m_{k+1}\left(-s_{k}\right)$, which is not required in the CTR algorithm. In this example, the extra expense of evaluating $m_{k+1}\left(-s_{k}\right)$ does not lead to larger trust-region radii in the RTR algorithm compared with CTR. Therefore, in this example RTR requires slightly more PDE solves than does CTR. See Table 5.2.

This small example can also be solved using a tensor-product discretization. We compare the solution from our adaptive approach with the solution from a level 7 tensor-product discretization. Figure 5.2 depicts the absolute pointwise control error. The relative $L^{2}(D)$-error in the controls is $5.0 \cdot 10^{-4}$ and the relative error in the final objective function values is $4.8 \cdot 10^{-5}$.

5.2. Optimal control of steady Burgers' equation. Our second example is the optimal control of Burgers' equation. In the deterministic case, this optimal control problem is analyzed in [63], and it is a special case of deterministic flow control problems discussed in [29].

Let $D=(0,1), \alpha>0$, and $\bar{w} \in L^{2}(D)$ be given. Furthermore, define the random coefficients

$$
\nu(y)=10^{y_{1}-2}, \quad f(y, x)=\frac{y_{2}}{100}, \quad d_{0}(y)=1+\frac{y_{3}}{1000}, \quad \text { and } \quad d_{1}(y)=\frac{y_{4}}{1000},
$$

where the random variables $y=\left(y_{1}, y_{2}, y_{3}, y_{4}\right)$ are uniformly distributed in $\Gamma=$ $[-1,1]^{4}$. The optimal control problem is given by

Copyright (c) by SIAM. Unauthorized reproduction of this article is prohibited. 


$$
\min \frac{1}{2} \int_{\Gamma} \rho(y) \int_{0}^{1}(u(y, x ; z)-\bar{w})^{2} \mathrm{~d} x \mathrm{~d} y+\frac{\alpha}{2} \int_{0}^{1} z^{2}(x) \mathrm{d} x,
$$

where $u(y, x ; z)$ solves

$$
\begin{array}{rr}
-\nu(y) \partial_{x x} u(y, x)+u(y, x) \partial_{x} u(y, x)=f(y, x)+z(x), & y \in \Gamma, x \in(0,1), \\
u(y, 0)=d_{0}(y), \quad u(y, 1)=d_{1}(y), & y \in \Gamma .
\end{array}
$$

For $y \in \Gamma$, the random viscosity parameter is the interval $\nu(y) \in\left[\nu_{\min }, \nu_{\max }\right] \equiv$ $\left[10^{-3}, 10^{-1}\right]$. The value of $\nu(y)$ has a significant impact on the solution of the nocontrol $(z=0)$ solution of Burgers' equation.

5.2.1. The infinite-dimensional problem. We can extend the problem setting of deterministic optimal control problems governed by Burgers' equation to fit (5.10), (5.11) into the abstract setting of section 2. We sketch the main steps, which are based on [63] and [1,32].

Using the construction in [62, Lem. 2.2, p. 71], we can show that there exists a function $u_{d} \in C_{\rho}^{0}\left(\Gamma ; H^{1}(0,1)\right)$ with $u_{d}(y, 0)=d_{0}(y), u_{d}(y, 1)=d_{1}(y)$ and $\left\|u_{d}(y, \cdot)\right\|_{L^{2}(0,1)} \leq \nu_{\min } / 2$ for all $y \in \Gamma$. Moreover, $\Gamma \ni y \mapsto u_{d}(y, \cdot) \in H^{1}(0,1)$ is continuously Fréchet differentiable. The solution of $(5.11)$ is $u(y, x)=u_{0}(y, x)+u_{d}(y, x)$, where $u_{0}$ solves Burgers' equation with homogeneous boundary conditions.

Let $\mathcal{V}=H_{0}^{1}(0,1), \mathcal{Z}=L^{2}(0,1)$, and for $y \in \Gamma$ define $\mathbf{A}(y) \in \mathcal{L}\left(\mathcal{V}, \mathcal{V}^{*}\right), \mathbf{B}(y) \in$ $\mathcal{L}\left(\mathcal{Z}, \mathcal{V}^{*}\right), \mathbf{b}(y) \in \mathcal{V}^{*}$ via (we use ' for differentiation with respect to $x$ )

$$
\begin{aligned}
\langle\mathbf{A}(y) u, \phi\rangle_{\mathcal{V}^{*}, \mathcal{V}} & =\int_{0}^{1} \nu(y) u^{\prime}(x) \phi^{\prime}(x)+u_{d}(y, x) u^{\prime}(x) \phi(x)+u_{d}^{\prime}(y, x) u(x) \phi(x) \mathrm{d} x, \\
\langle\mathbf{B}(y) z, \phi\rangle_{\mathcal{V}^{*}, \mathcal{V}} & =-\int_{0}^{1} z(x) \phi(x) \mathrm{d} x \\
\langle\mathbf{b}(y), \phi\rangle_{\mathcal{V}^{*}, \mathcal{V}} & =\int_{0}^{1} \nu(y) u_{d}^{\prime}(y, x) \phi^{\prime}(x)+u_{d}(y, x) u_{d}^{\prime}(y, x) \phi(x)-f(x, y) \phi(x) \mathrm{d} x
\end{aligned}
$$

for all $u, \phi \in \mathcal{V}$ and all $z \in \mathcal{Z}$, and define the nonlinear operator $u \mapsto \mathbf{N}(u) \in \mathcal{V}^{*}$ by

$$
\langle\mathbf{N}(u), \phi\rangle_{\mathcal{V}^{*}, \mathcal{V}}=\int_{0}^{1} u^{\prime}(x) u(x) \phi(x) \mathrm{d} x
$$

The weak solution $u$ of $(5.11)$ is $u=u_{0}+u_{d}$, where $u_{0}$ solves

$$
\mathbf{A}(y) u_{0}(y)+\mathbf{N}\left(u_{0}(y)\right)+\mathbf{B}(y) z+\mathbf{b}(y)=0 \quad \text { in } \mathcal{V}^{*} .
$$

By applying $[27$, Thm. 2.3, p. 291], [62, Thm. 2.10, p. 74] we can show that there exists at least one solution of (5.12) and for $\nu_{\min }>\nu_{0}\left(f, z, d_{1}, d_{2}\right)$ there exists a unique solution $u_{0}(y ; z)$ of (5.12). Moreover, there exists $c>0$ independent of $y \in \Gamma$ and $z \in \mathcal{Z}$ such that

$$
\left\|u_{0}(y ; z)\right\|_{H_{0}^{1}(0,1)} \leq c\left(\|z\|_{\mathcal{Z}}+1\right) \quad \text { for all } z \in \mathcal{Z} \text { and all } y \in \Gamma .
$$

The nonlinear operator $\mathcal{V} \ni u \mapsto \mathbf{N}(u) \in \mathcal{V}^{*}$ is Fréchet differentiable with derivative

$$
\left\langle\mathbf{N}^{\prime}(u) v, \phi\right\rangle_{\mathcal{V}^{*}, \mathcal{V}}=\int_{0}^{1}\left(u^{\prime}(x) v(x)+v^{\prime}(x) u(x)\right) \phi(x) \mathrm{d} x .
$$

Copyright (C) by SIAM. Unauthorized reproduction of this article is prohibited. 
Using the techniques in [63, p. 63], we can show that for every $y \in \Gamma$ and every $u \in H_{0}^{1}(0,1)$ the operator $\mathbf{A}(y)+\mathbf{N}^{\prime}(u)$ is continuously invertible. For $\nu_{\min }>\nu_{0}(u)$, there exists a constant $c>0$ such that $\left\|\left(\mathbf{A}(y)+\mathbf{N}^{\prime}(u)\right)^{-1}\right\| \leq c$ for all $y \in \Gamma[32$, Thm. 3.3]. Moreover, for every $u_{0} \in H_{0}^{1}(0,1)$ and $z \in L^{2}(0,1)$ the map $\Gamma \ni y \mapsto$ $\mathbf{A}(y) u_{0}+\mathbf{N}\left(u_{0}\right)+\mathbf{B}(y) z+\mathbf{b}(y) \in \mathcal{V}^{*}$ is continuously Fréchet differentiable. Therefore, the implicit function theorem implies that $u_{0}(\cdot ; z) \in C^{1}(\Gamma, \mathcal{V})$ for all $z \in \mathcal{Z}$.

5.2.2. Discretization and numerical results. We discretized Burgers' equation in space using continuous piecewise linear finite elements on a uniform mesh of $N=2000$ elements. Our high-fidelity stochastic collocation discretization uses a level 8 isotropic Smolyak sparse grid built on 1D Clenshaw-Curtis interpolation knots. (We could also have used Gauss-Patterson knots as in the previous example.) To solve the discretized nonlinear system at each collocation point, we use Newton's method with a backtracking line search. For this example, $\alpha=10^{-3}$ and $\bar{w} \equiv 1$. The results of the optimization are depicted in Figures 5.3 and 5.4. The left image in Figure 5.3 highlights the difference between the optimal controls for the stochastic problem (black) and the deterministic substitute problem with $y$ replaced by $E[y]=0$ (red). The right image shows the expected value of the optimal state with one and two standard deviations added and subtracted. Figure 5.4 displays the final adapted sparse grid index
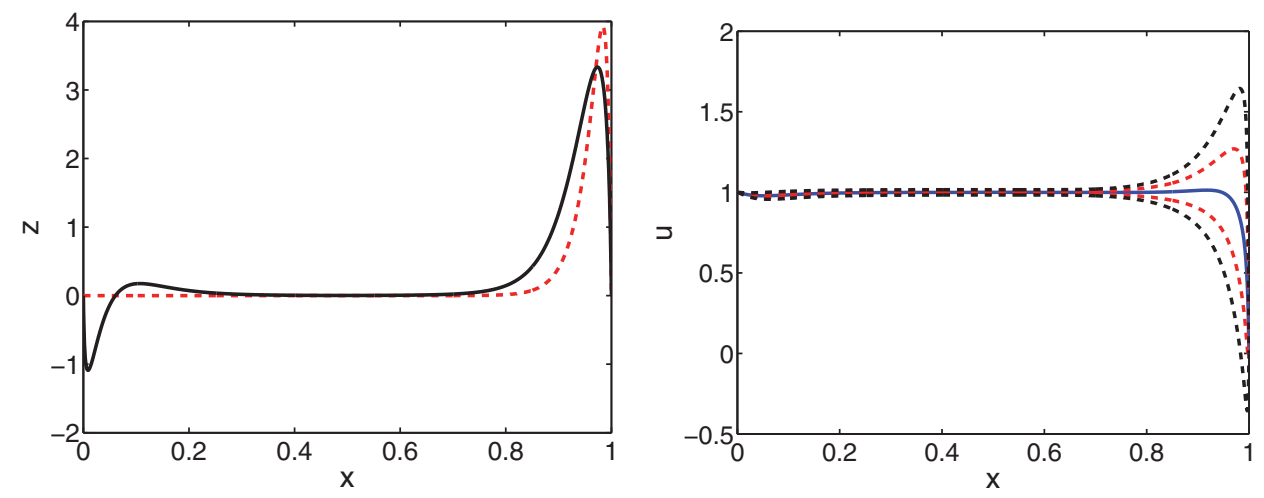

FIG. 5.3. Left: The optimal control for the stochastic problem (black solid line) and and mean value control (i.e., $y$ replaced by $E[y]=0$, red dashed line). Right: The expected value of the optimal state plus/minus one and two standard deviations.
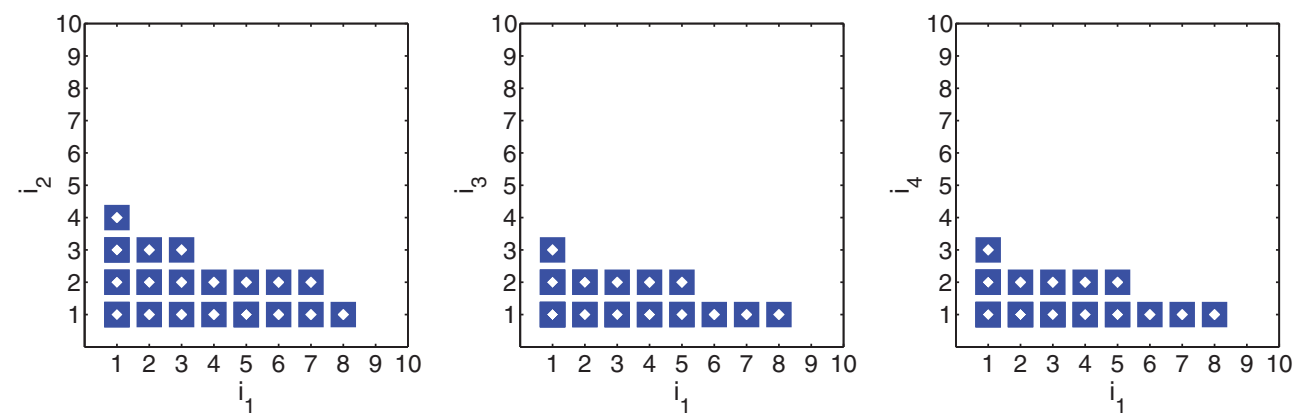

FIG. 5.4. Comparison of the adapted index set. The left figure compares dimension 1 with dimension 2 , the middle figure compares dimension 1 with dimension 3 , and the right figure compares dimension 1 with dimension 4. 
TABLE 5.3

Iteration history of the RTR algorithm applied to Example 5.2. Here $k$ is the iteration counter, $\widehat{J}\left(z_{k}\right)$ is the objective function value, $\left\|\nabla \widehat{J}_{\mathcal{I}}\left(z_{k}\right)\right\|_{\mathcal{Z}}$ is the model gradient norm value, $\left\|s_{k}\right\| \mathcal{Z}$ is the step size, $\Delta_{k}$ is the trust-region radius, $C G$ is the number of $C G$ iterations, Adaptive is the number of sparse grid adaptation iterations, and $Q$ is the number of collocation points.

\begin{tabular}{cccccccc}
\hline$k$ & $\widehat{J}\left(z_{k}\right)$ & $\left\|\nabla \widehat{J}_{\mathcal{I}}\left(z_{k}\right)\right\|_{\mathcal{Z}}$ & $\left\|s_{k}\right\|_{\mathcal{Z}}$ & $\Delta_{k}$ & $\mathrm{CG}$ & Adaptive & $Q$ \\
\hline 0 & $8.310663 \mathrm{e}-03$ & $9.848394 \mathrm{e}-03$ & - & 1000 & - & - & 1 \\
1 & $6.777993 \mathrm{e}-03$ & $1.231346 \mathrm{e}-03$ & 0.5080726 & 2500 & 4 & 5 & 29 \\
2 & $6.738253 \mathrm{e}-03$ & $9.142368 \mathrm{e}-03$ & 0.3478721 & 2500 & 7 & 0 & 29 \\
3 & $6.334151 \mathrm{e}-03$ & $1.068670 \mathrm{e}-03$ & 0.2465402 & 5000 & 4 & 0 & 29 \\
4 & $6.289515 \mathrm{e}-03$ & $5.755139 \mathrm{e}-05$ & 0.1471733 & 5000 & 7 & 3 & 51 \\
5 & $6.288987 \mathrm{e}-03$ & $3.462104 \mathrm{e}-06$ & 0.0234743 & 5000 & 7 & 3 & 87 \\
6 & $6.288986 \mathrm{e}-03$ & $2.905453 \mathrm{e}-07$ & 0.0014251 & 5000 & 5 & 5 & 137 \\
\hline
\end{tabular}

TABLE 5.4

The total number of outer iterations (Iter), adaptive steps (Adaptive), and PDE solves (PDE) and final number of collocation points used for the model ( $Q$ ) for the three algorithms applied to Example 5.2. The fourth column contains the ratio of the total number of PDE solves for Newton-CG versus the total number of PDE solves for the other algorithms (Ratio).

\begin{tabular}{lccrrr}
\hline Algorithm & Iter & Adaptive & PDE & $Q$ & Ratio \\
\hline Newton-CG & 4 & 0 & 806,461 & 7,537 & 1.00 \\
CTR & 6 & 17 & 59,847 & 137 & 13.48 \\
RTR & 6 & 17 & 60,297 & 137 & 13.37 \\
\hline
\end{tabular}

set. Each image is the projection of the index set on to the 2D planes corresponding to the multi-indices $\left(i_{1}, i_{2}\right),\left(i_{1}, i_{3}\right)$, and $\left(i_{1}, i_{4}\right)$. From these images, one can clearly see that the $i_{1}$ direction is significantly more important than the other directions.

Table 5.3 displays the iteration history for the RTR algorithm, while Table 5.4 contains a comparison of Newton-CG, the CTR algorithm, and the RTR algorithm. As in the previous example, applying the adaptive algorithm is significantly faster than using a fixed stochastic collocation discretization. For both CTR and RTR, all iterations are successful, and the same trust-region radii are generated. In this case, RTR requires more PDE solves than does CTR, because RTR requires the evaluation of a new model at the previous iterate, $m_{k+1}\left(-s_{k}\right)$.

5.3. Optimal control of stochastic Helmholtz equation. Our final example is motivated by the optimal control of direct field acoustic testing (DFAT) [41]. An important objective in DFAT is to accurately shape sound pressure fields in a region of interest by using acoustic sources, such as loudspeakers, located away from and directed at the region of interest. When the refractive index of the medium in the region of interest is random, we may assume that the governing physics are modeled by the stochastic Helmholtz equation, as derived, for example, in [44, 31, 42]. The stochasticity is often represented by a Karhunen-Loéve (KL) expansion of the refractive index.

In [68], the authors present numerical methods for solving the Helmholtz equation with stochastic boundary data. For optimal control problems governed by the deterministic Helmholtz equation, see, for example, [9] and the references therein. The optimal control of the stochastic Helmholtz equation is studied in [16] in the context of controlling radiated engine noise. The solution of an inverse problem governed by the Helmholtz equation is discussed, for example, in [19].

We consider an idealized DFAT example in two spatial dimensions, where the domain is $D=(-5,5)^{2}$. The goal of this control problem is to match the wave 
pressure $u$ to a desired wave pressure $\bar{w} \in L^{2}(D ; \mathbb{C})$ in the disk $D_{R} \subset D, D_{R}:=$ $\left\{x \in D:\|x\|_{2} \leq R\right\}$, with $R \in(0,5)$. For simplicity, rather than applying discrete acoustic controls, we apply a distributed control in the annulus $D_{C}$ exterior to $D_{R}$, $D_{C}=\left\{x \in D: R+d \leq\|x\|_{2} \leq R+d+w\right\}$, where $d, w>0$ are such that $R+d+$ $w<5$. Figure 5.5 depicts the experimental setup.

The optimal control problem is given by

$\min _{z \in L^{2}(D ; \mathbb{C})} \frac{1}{2} \int_{\Gamma} \rho(y) \int_{D_{R}}(u(z ; y, x)-\bar{w}(x)) \overline{(u(z ; y, x)-\bar{w}(x))} \mathrm{d} x \mathrm{~d} y+\frac{\alpha}{2} \int_{D_{c}} z(x) \overline{z(x)} \mathrm{d} x$,

where $u(z ; y, \cdot)=u(y, \cdot) \in H^{1}(D ; \mathbb{C})$ for all $y \in \Gamma=[-\sqrt{3}, \sqrt{3}]^{M}$ solves

$$
-\Delta u(y, x)-k^{2}(1+\sigma \epsilon(y, x))^{2} u(y, x)=z(x) \quad \forall(y, x) \in \Gamma \times D
$$

with Robin boundary conditions

$$
\frac{\partial u}{\partial n}(y, x)=i k u(y, x) \quad \forall(y, x) \in \Gamma \times \partial D .
$$

In (5.14), $u$ is the wave pressure, $k>0$ is the wave number, and $(1+\sigma \epsilon)$ denotes the stochastic refractive index [44,31, 42]. Assuming that the physical domain is sufficiently large, the Sommerfeld radiation condition holds. The boundary conditions (5.15) are a first-order approximation of the Sommerfeld condition. The desired complex-valued wave pressure is given by the plane-wave expression

$$
\bar{w}(x)=\exp \left(i\left((k \cos \theta) x_{1}+(k \sin \theta) x_{2}\right)\right)
$$

for a fixed angle of propagation $\theta$. The real and imaginary components of the desired wave pressure are plotted for $\theta=\pi / 4$ and $k=10$ in Figure 5.6.

Similar to [42], we assume that the stochastic refractive index satisfies

$$
\sigma \ll 1, \quad E[\epsilon(\cdot, x)]=0, \quad \text { and } \quad E[\epsilon(\cdot, x) \epsilon(\cdot, \zeta)]=C(\|x-\zeta\|),
$$

where $C$ denotes a covariance function. In [31], the covariance function $C$ is chosen to be exponential, Gaussian, or Kolmogorov. For this numerical example, we choose $C$ to be an instance of the Matérn covariance functions,
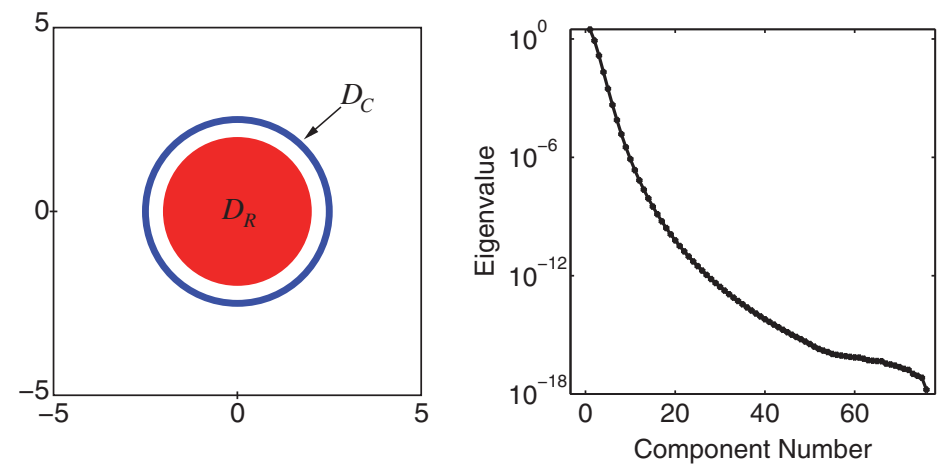

FIG. 5.5. Left: Control region, $D_{C}$, and region of interest, $D_{R}$. Right: Eigenvalues of the Matérn covariance function.

Copyright $\odot$ by SIAM. Unauthorized reproduction of this article is prohibited. 

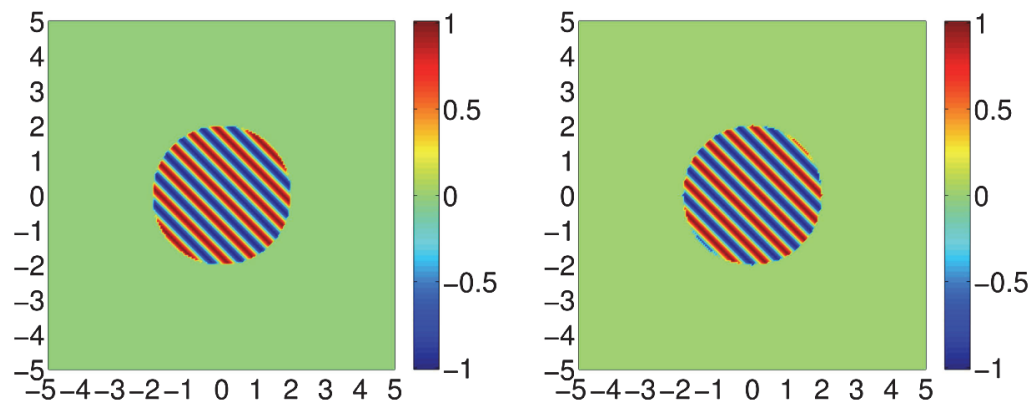

FIG. 5.6. Real (left) and imaginary (right) parts of the desired state, $\bar{w}$.

$$
C(r)=C_{\nu}(r):=\frac{2^{1-\nu}}{\Gamma(\nu)}\left(\frac{2 \sqrt{\nu} r}{\ell}\right)^{\nu} \mathbf{K}_{\nu}\left(\frac{2 \sqrt{\nu} r}{\ell}\right) .
$$

Here, $\boldsymbol{\Gamma}(\nu)$ is the gamma function, and $\mathbf{K}_{\nu}$ is the modified Bessel function of the third kind [59]. The parameter $\nu>0$ controls the smoothness of the underlying random process, and the parameter $\ell>0$ represents the correlation length of the random process [59]. We have chosen the Matérn class because it contains both the exponential $\left(\nu=\frac{1}{2}\right)$ and the squared exponential covariance functions $(\nu \rightarrow \infty)$.

We approximate the refractive index using a truncated KL expansion of order $M$,

$$
\epsilon(y, x) \approx \sum_{m=1}^{M} \epsilon_{m}(x) y_{m} .
$$

The coefficients $\epsilon_{m}: D \rightarrow \mathbb{R}$ for $m=1, \ldots, M$ and $y_{m}$ are uncorrelated random variables with zero mean and unit variance for $m=1, \ldots, M$. We have chosen $y_{m}$ for $m=1, \ldots, M$ to be independent and uniformly distributed on the interval $\Gamma_{m}=[-\sqrt{3}, \sqrt{3}]$.

5.3.1. The infinite-dimensional problem. We will replace the complex functions $u=u_{r}+i u_{i}$ with $u_{r}, u_{i} \in H^{1}(D)$ and $z=z_{r}+i z_{i}$ with $z_{r}, z_{i} \in L^{2}(D)$ with their vector equivalents $u=\left(u_{r}, u_{i}\right)^{\top} \in H^{1}(D) \times H^{1}(D)$ and $z=\left(z_{r}, z_{i}\right)^{\top} \in L^{2}(D) \times L^{2}(D)$, respectively. Therefore, we define $\mathcal{V}=H^{1}(D) \times H^{1}(D)$ and $\mathcal{Z}=L^{2}(D) \times L^{2}(D)$. Furthermore, we define the (parametrized) linear operators $A_{1}(y) \in \mathcal{L}\left(H^{1}(D), H^{1}(D)^{*}\right)$ for $y \in \Gamma, A_{2} \in \mathcal{L}\left(H^{1}(D), H^{1}(D)^{*}\right)$, and $B \in \mathcal{L}\left(L^{2}(D), H^{1}(D)^{*}\right)$ by the relationships

$$
\begin{aligned}
\left\langle A_{1}(y) u, v\right\rangle_{H^{1}(D)^{*}, H^{1}(D)} & =\int_{D} \nabla u(x) \cdot \nabla v(x)-k^{2}(1+\sigma \epsilon(y, x))^{2} u(x) v(x) \mathrm{d} x, \\
\left\langle A_{2} u, v\right\rangle_{H^{1}(D)^{*}, H^{1}(D)} & =k \int_{\partial D} u(x) v(x) \mathrm{d} x, \\
\langle B z, v\rangle_{H^{1}(D)^{*}, H^{1}(D)} & =-\int_{D} z(x) v(x) \mathrm{d} x .
\end{aligned}
$$

Substituting $u=u_{r}+i u_{i}$ and $z=z_{r}+i z_{i}$ into the weak form of (5.14), (5.15) and factoring the real and imaginary parts, we obtain the linear system of equations

$$
\left(\begin{array}{cc}
A_{1}(y)+A_{2} & A_{1}(y)-A_{2} \\
-A_{1}(y)+A_{2} & A_{1}(y)+A_{2}
\end{array}\right)\left(\begin{array}{l}
u_{r}(y) \\
u_{i}(y)
\end{array}\right)+\left(\begin{array}{cc}
B & B \\
-B & B
\end{array}\right)\left(\begin{array}{c}
z_{r} \\
z_{i}
\end{array}\right)=0 .
$$

Copyright $@$ by SIAM. Unauthorized reproduction of this article is prohibited. 
This is an equation of the form $\mathbf{A}(y) u(y)+\mathbf{B} z=0$ with $\mathbf{A}(y) \in \mathcal{L}\left(\mathcal{V}, \mathcal{V}^{*}\right)$ for all $y \in \Gamma$ and $\mathbf{B} \in \mathcal{L}\left(\mathcal{Z}, \mathcal{V}^{*}\right)$, it fits the form of (2.2). Because $\mathbf{A}(y)$ is not coercive, we have not yet been able to verify the assumptions of section 2 .

In this vector notation, we rewrite (5.13) as

$$
\min _{z \in \mathcal{Z}} \frac{1}{2} \int_{\Gamma} \rho(y)\langle\mathbf{C}(u(z ; y)-\bar{v}), u(z ; y)-\bar{v}\rangle_{\mathcal{V} *, \mathcal{V}} \mathrm{d} y+\frac{\alpha}{2}\langle\mathbf{R} z, z\rangle_{\mathcal{Z}^{*}, \mathcal{Z}},
$$

where $u(z ; y)=\left(u_{r}(y), u_{i}(y)\right)^{\top} \in \mathcal{V}$ for all $y \in \Gamma$ solves $(5.16), \bar{v}=(\Re(\bar{w}), \Im(\bar{w}))^{\top} \in$ $L^{2}(D) \times L^{2}(D), \mathbf{C} \in \mathcal{L}\left(\mathcal{V}, \mathcal{V}^{*}\right)$ satisfies

$$
\langle\mathbf{C} u, v\rangle_{\mathcal{V}^{*}, \mathcal{V}}=\int_{D_{R}} u_{r}(x) v_{r}(x)+u_{i}(x) v_{i}(x) \mathrm{d} x,
$$

and $\mathbf{R} \in \mathcal{L}\left(\mathcal{Z}, \mathcal{Z}^{*}\right)$ satisfies

$$
\langle\mathbf{R} z, w\rangle_{\mathcal{Z}^{*}, \mathcal{Z}}=\int_{D_{c}} z_{r}(x) w_{r}(x)+z_{i}(x) w_{i}(x) \mathrm{d} x .
$$

5.3.2. Discretization and numerical results. We discretize (5.16) in space using continuous Q1 finite elements on a uniform mesh of $200 \times 200$ quadrilaterals. The high-fidelity collocation discretization is built on 1D Clenshaw-Curtis interpolation knots. The radius of the region of interest is $R=2$, the distance to the control annulus is $d=0.5$, and the width of the control annulus is $w=0.1$. Furthermore, the wave number is $k=10$, the acoustic perturbation parameter is $\sigma=0.1$, the control penalty parameter is $\alpha=10^{-4}$, and the desired wave angle is $\theta=\pi / 4$. The Matérn function parameters are $\nu=\frac{11}{2}$ and $\ell=2$. The number of random variables is given by the order $M$ of the KL expansion of the refractive index. Here we consider high-dimensional stochastic problems with $M \in\{20,30,40\}$.

Table 5.5 displays the iteration history of the CTR algorithm for a 20-dimensional stochastic problem, $M=20$. Table 5.6 gives the computational cost as the stochastic dimension increases from $M=20$ to $M=40$. We note that for $M=20$ the evaluation of the objective function is based on a high-fidelity collocation grid comprising 120,401 nodes. In contrast, all other optimization quantities are evaluated on much smaller, adapted grids. The final (i.e., largest) adapted grid contains only 1,405 nodes. In other words, the solution of the optimization problem is fully dominated by the evaluation

TABLE 5.5

Iteration history of the CTR algorithm applied to the Helmholtz example with $M=20$. Here $k$ is the iteration counter, $\widehat{J}\left(z_{k}\right)$ is the objective function value, $\left\|\nabla \widehat{J}_{\mathcal{I}}\left(z_{k}\right)\right\|_{\mathcal{Z}}$ is the model gradient norm value, $\left\|s_{k}\right\|_{\mathcal{Z}}$ is the step size, $\Delta_{k}$ is the trust-region radius, $C G$ is the number of CG iterations, Adaptive is the number of sparse grid adaptation iterations, and $Q$ is the number of collocation points.

\begin{tabular}{c|ccrcccc}
\hline$k$ & $\widehat{J}\left(z_{k}\right)$ & $\left\|\nabla \widehat{J}_{\mathcal{I}}\left(z_{k}\right)\right\|_{\mathcal{Z}}$ & $\left\|s_{k}\right\|_{\mathcal{Z}}$ & $\Delta_{k}$ & $\mathrm{CG}$ & Adaptive & $Q$ \\
\hline 0 & $5.948729 \mathrm{e}+00$ & $1.014288 \mathrm{e}-01$ & - & $1.00 \mathrm{e}+03$ & - & - & 1 \\
1 & $7.936557 \mathrm{e}+00$ & $1.012872 \mathrm{e}-01$ & $1.06 \mathrm{e}+02$ & $5.28 \mathrm{e}+01$ & 4 & 6 & 57 \\
2 & $5.740645 \mathrm{e}+00$ & $3.158975 \mathrm{e}-02$ & $5.28 \mathrm{e}+01$ & $5.28 \mathrm{e}+01$ & 1 & 4 & 91 \\
3 & $5.349043 \mathrm{e}+00$ & $2.899215 \mathrm{e}-03$ & $3.41 \mathrm{e}+01$ & $1.32 \mathrm{e}+02$ & 3 & 16 & 197 \\
4 & $5.338680 \mathrm{e}+00$ & $2.193334 \mathrm{e}-03$ & $4.23 \mathrm{e}+00$ & $3.30 \mathrm{e}+02$ & 3 & 2 & 209 \\
5 & $5.335895 \mathrm{e}+00$ & $1.250368 \mathrm{e}-04$ & $2.56 \mathrm{e}+00$ & $8.25 \mathrm{e}+02$ & 2 & 25 & 367 \\
6 & $5.335868 \mathrm{e}+00$ & $5.244318 \mathrm{e}-05$ & $4.79 \mathrm{e}-01$ & $2.06 \mathrm{e}+03$ & 4 & 18 & 537 \\
7 & $5.335867 \mathrm{e}+00$ & $5.273816 \mathrm{e}-06$ & $6.50 \mathrm{e}-02$ & $5.00 \mathrm{e}+03$ & 2 & 37 & 829 \\
8 & $5.335867 \mathrm{e}+00$ & $6.432970 \mathrm{e}-07$ & $2.99 \mathrm{e}-02$ & $5.00 \mathrm{e}+03$ & 5 & 59 & 1405 \\
\hline
\end{tabular}

Copyright (c) by SIAM. Unauthorized reproduction of this article is prohibited. 
TABLE 5.6

Computational cost of the CTR algorithm applied to the Helmholtz example with $M \in$ $\{20,30,40\}$. Here $M$ is the stochastic dimension, CTR it is the number of iterations of the CTR algorithm, PDE solves is the total number of forward and adjoint PDE solves, Hi-fi grid is the number of collocation points in the high-fidelity stochastic grid, Adpt grid is the number of collocation points in the final adapted grid, Nodes/Cores is the numbers of compute nodes and the numbers of cores per node used for each test case, and Time (h) is the computational time for the trust-region algorithm, measured in hours.

\begin{tabular}{l|rrrrrr}
\hline M & CTR it & PDE solves & Hi-fi grid & Adpt grid & Nodes/Cores & Time (h) \\
\hline 20 & 9 & $1,136,784$ & 120,401 & 1,405 & $64 / 8$ & 7.8 \\
30 & 9 & $5,316,021$ & 582,801 & 849 & $128 / 8$ & 14.0 \\
40 & 9 & $16,327,120$ & $1,804,001$ & 1,445 & $256 / 6$ & 25.2 \\
\hline
\end{tabular}

of the high-fidelity objective function. All remaining optimization steps are essentially free.

Specifically, for the $M=20$ case our algorithm reports 9 objective function evaluations, each with 120,401 forward-PDE solves, combining to 1,083,609 PDE solves. This is $95 \%$ of the total of 1,136,784 PDE solves reported in Table 5.6. Similar observations hold for the cases $M=30$ and $M=40$. More fundamentally, Tables 5.5 and 5.6 demonstrate that the adaptive trust-region algorithm enables the solution of high-dimensional stochastic PDE optimization problems. The adaptive scheme efficiently detects dominant stochastic directions and reduces computational cost.

Figures 5.7 and 5.8 show the optimization results. An important observation from Figure 5.7 is that the computed optimal controls for the stochastic problem are
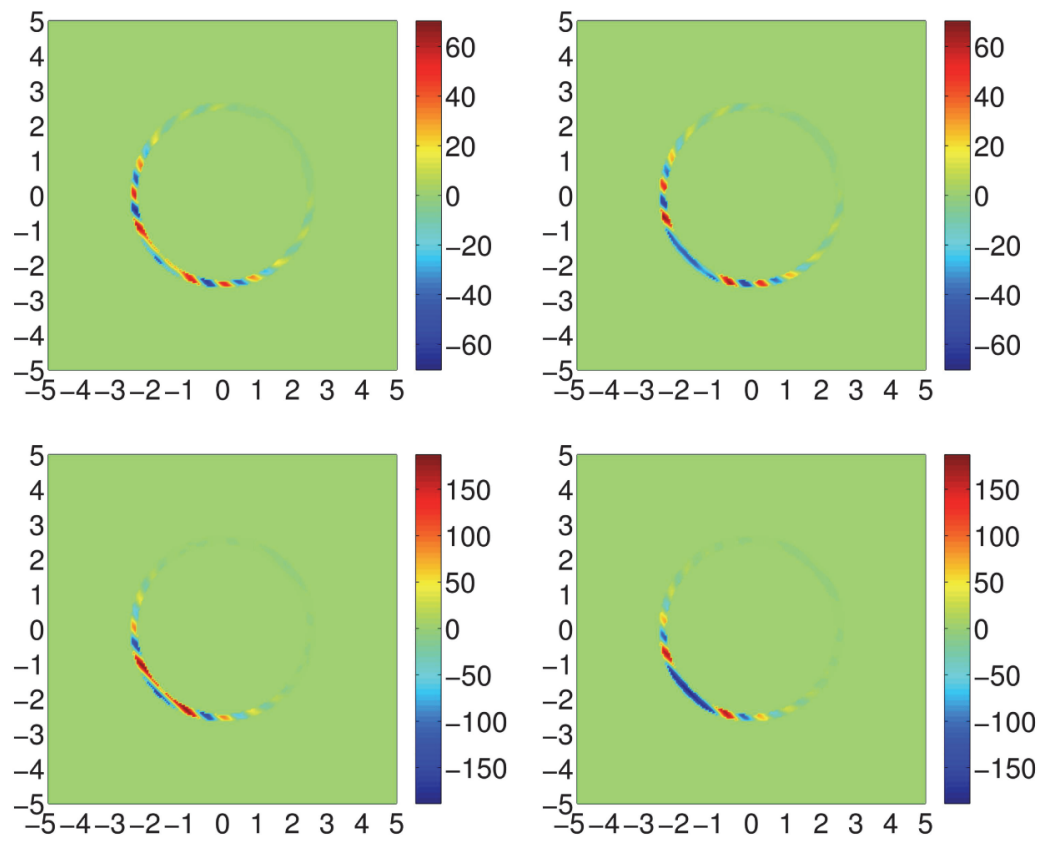

FIG. 5.7. Top: Optimal controls for the stochastic problem, including real (left) and imaginary (right) components of the optimal solution. Bottom: Optimal controls for the deterministic substitute problem, where $y$ is replaced by $E[y]=0$, including real (left) and imaginary (right) components of the solution. Note that the controls for the stochastic problem are about three times smaller in magnitude than the controls for the deterministic substitute problem. 

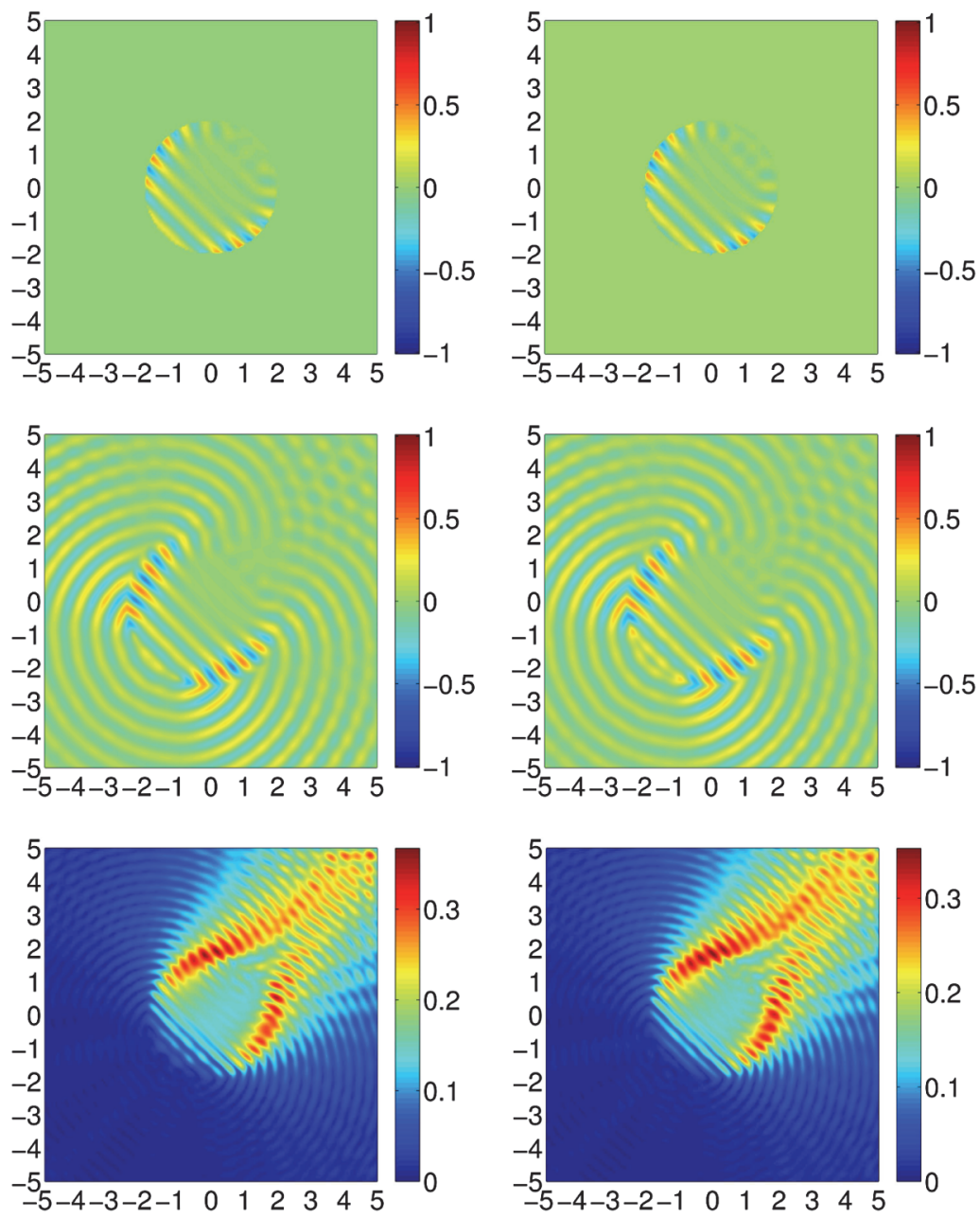

FIG. 5.8. Top: Real (left) and imaginary (right) parts of the expected value of the optimal state, restricted to the region of interest. Middle: Real (left) and imaginary (right) parts of the expected value of the optimal state, over the entire computational domain. Bottom: Real (left) and imaginary (right) parts of the standard deviation of the optimal state. Note that the standard deviation is large in the top-right quadrant of the domain. Accordingly, the match to the desired wave pressure is worse in this region; see top row.

significantly smaller in magnitude than the controls obtained by solving the deterministic substitute problem, where $E[y]=0$. We also note that the achieved value of the stochastic objective function is approximately 5.3 in the first case and 7.9 in the second, a difference of $50 \%$. Figure 5.8 demonstrates that the chosen acoustic perturbation and Matérn covariance parameters yield a large amount of randomness in the refraction index. Consequently, the expected value of the optimal state is not a great match to the desired plane-wave profile given in Figure 5.6. The mismatch is particularly noticeable in the top-right quadrant.

Figure 5.9 shows the probability density function (PDF; left image) and the cumulative distribution function (CDF; right image) of the quantity $\mathcal{J}(y, z)=\langle\mathbf{C}(u(y ; z)-$ $\bar{v}), u(y ; z)-\bar{v}\rangle_{\mathcal{V}^{*}, \mathcal{V}}$. The blue lines correspond to the PDF and $\mathrm{CDF}$ of $\mathcal{J}(y, z)$ at the optimal control for the stochastic optimization problem, and the red lines correspond 

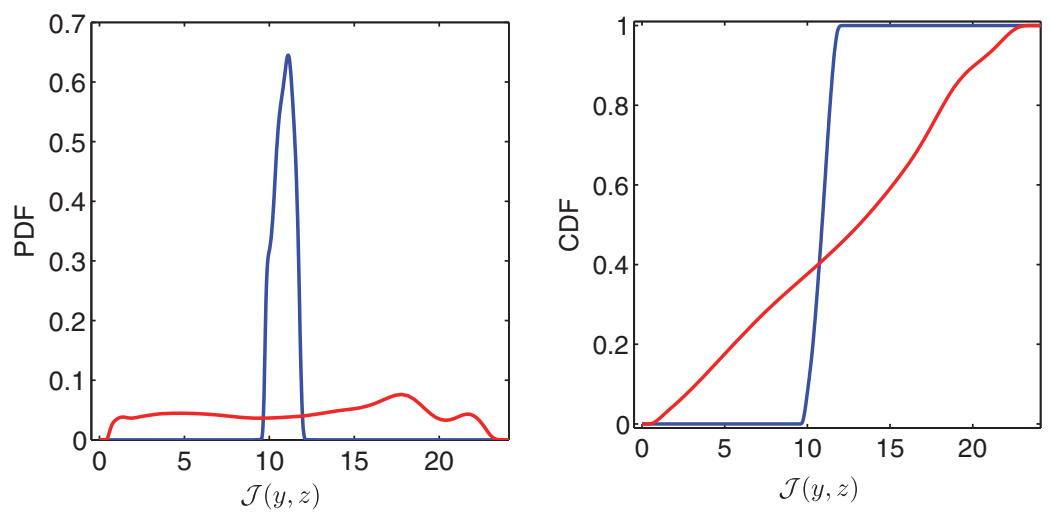

FIG. 5.9. Left: PDF of the quantity $\mathcal{J}(y, z)=\langle\mathbf{C}(u(y ; z)-\bar{v}), u(y ; z)-\bar{v}\rangle_{\mathcal{V}^{*}, \mathcal{V}}$ for the optimal control from the stochastic problem (blue line) and the optimal control from the deterministic substitute problem (red line). Right: $C D F$ of $\mathcal{J}(y, z)$. The PDF and CDF plots clearly indicate that the optimal control for the stochastic problem significantly reduces the variance associated with $\mathcal{J}(y, z)$.

to the PDF and CDF of $\mathcal{J}(y, z)$ at the optimal control for the deterministic substitute problem, where $y \in \Gamma$ is replaced by $E[y]=0$. The PDFs and CDFs were computed using Monte Carlo sampling of the stochastic Helmholtz equation. The CDFs are sample-average approximations of the true CDFs, whereas the PDFs are kernel smoothed approximations of the true PDFs. We increased the Monte Carlo sample size by 1,000 samples until the observed changes in the $L^{\infty}$-norm of the computed CDFs were less than $10^{-3}$. This resulted in a Monte Carlo sample size of 25,000 . These figures demonstrate that the optimal control for the stochastic problem significantly reduces the variance associated with $\mathcal{J}(y, z)$ when compared with the deterministic control. Therefore, the control for the stochastic problem results in a more predictable mismatch between the solution to stochastic Helmholtz, $u(y ; z)$, and the desired profile $\bar{w}$.

6. Conclusions. We have introduced a trust-region algorithm with adaptive sparse-grid collocation for the numerical solution of optimization problems governed by PDEs with uncertain coefficients. The algorithm exploits anisotropy in the stochastic nature of the PDE to adaptively build the stochastic collocation space. By enforcing a gradient consistency condition, the algorithm is provably globally first-order convergent. Currently, the gradient consistency condition is implemented by using an error indicator. Although there is no proof that the gradient consistency holds for general PDE-constrained optimization problems, numerical results suggest that the heuristic bounds presented in this paper work well for many problems. Furthermore, the numerical results demonstrate a dramatic reduction in the number of PDE solves required to obtain a minimizer when compared with the number of PDE solves required by a Newton-CG method applied to the high-fidelity sparse-grid discretization.

The computational work of our algorithm is dominated by the high-fidelity objective function evaluations. We may be able to further reduce the total number of PDE solves by employing inexact objective-function evaluations. Trust-region algorithms with inexact objective-function evaluations have been proposed in [17], [21, sect. 10.6], [72]. The approach [17], [21, sect. 10.6] requires the error estimates that allow one to reduce the error in function evaluations below a prescribed level. Therefore, asymptotic error estimates do not fit into the framework of [17], [21, sect. 10.6]. In [72] 
a slightly different consistency condition for inexact objective functions is proposed in the context of adaptive mesh refinement for deterministic PDE-constrained optimization problems. A single mesh is adapted to generate both the inexact objective function as well as the model. For stochastic collocation discretizations, our initial numerical results involving inexact objective functions suggests that the adaptive refinement of the objective function and its model should be decoupled. In particular, joint refinement of the objective function and its model appears to result in a very large sparse grid during the first few iterations. Therefore, it is not yet clear how to extend the conditions and corresponding theory in [17], [21, sect. 10.6], [72] to our context.

We use the error indicator $\eta$ in (4.6) to implement the inexact gradient condition (4.3), (4.4). It is desirable to have an error estimator $\eta \geq C \sum_{\mathbf{i} \notin \mathcal{I}} \|\left(\Delta_{1}^{i_{1}} \otimes \cdots \otimes\right.$ $\left.\Delta_{M}^{i_{M}}\right)\left[\mathbf{B}^{*} p\right] \|_{\mathcal{Z}}$, which would allow us to rigorously implement (4.3), (4.4). In the context of solving single PDEs with random data, an adaptive scheme based on Taylor approximations has been proposed in [20], and an a posteriori error estimator has been proved. Use of this and other stochastic discretizations such as stochastic Galerkin [4] with the goal to derive an error estimator in the optimization context is another avenue for possible research.

Appendix A. Convergence proof. In this section, we prove that under Assumption 4.1, Algorithm 4.2 converges to a first-order critical point. First, we prove that the sequence of trust-region radii must converge to zero if the norm of the gradients is bounded away from zero. Second, we show that under these assumptions $\varrho_{k}$ and $\tilde{\varrho}_{k+1}$ converge to one. These results are then combined to prove that Algorithm 4.1 converges to a first-order critical point. Most results presented here follow the standard convergence proof for the basic trust-region algorithm provided in Theorem 4.10 in [43], although care must be taken to handle the RTR update.

Lemma A.1. Suppose there exists $\epsilon>0$ such that $\left\|\nabla m_{k}(0)\right\|_{\mathcal{Z}} \geq \epsilon$ for $k$ sufficiently large. Then the sequence of trust-region radii, $\left\{\Delta_{k}\right\}$, produced by Algorithm 4.2 satisfies

$$
\sum_{k=1}^{\infty} \Delta_{k}<\infty
$$

Proof. First notice that the result of the theorem holds if there are only a finite number of successful iterations because for sufficiently large $k, \Delta_{k+1} \leq \gamma_{1} \Delta_{k}$. Now, if there is an infinite sequence of successful iterations $\left\{k_{i}\right\}$, then for sufficiently large $i$ the fraction of Cauchy decrease condition (4.2) implies

$$
\begin{aligned}
J\left(z_{k_{i}}\right)-J\left(z_{k_{i+1}}\right) & \geq J\left(z_{k}\right)-J\left(z_{k+1}\right) \\
& \geq \eta_{0}\left(m_{k}(0)-m_{k}\left(s_{k}\right)\right) \\
& \geq \eta_{0} \kappa_{0}\left\|\nabla m_{k}(0)\right\|_{\mathcal{Z}} \min \left\{\Delta_{k}, \frac{\left\|\nabla m_{k}(0)\right\|_{\mathcal{Z}}}{\beta_{k}}\right\} \\
& \geq \eta_{0} \kappa_{0} \Delta_{k} \epsilon .
\end{aligned}
$$

This implies that $\sum_{i=1}^{\infty} \Delta_{k_{i}}<\infty$. Furthermore, for every unsuccessful iteration $k \notin\left\{k_{i}\right\}$, the trust-region radius satisfies $\Delta_{k} \leq \gamma_{1}^{k-k_{j}} \Delta_{k_{j}}$ where $k_{j} \in\left\{k_{i}\right\}$ is the largest index such that $k_{j}<k$. The convergence of geometric series and the above result imply that 


$$
\sum_{k \notin\left\{k_{i}\right\}} \Delta_{k} \leq \frac{1}{1-\gamma_{1}} \sum_{i=1}^{\infty} \Delta_{k_{i}} \text { and } \sum_{k=1}^{\infty} \Delta_{k} \leq\left(1+\frac{1}{1-\gamma_{1}}\right) \sum_{i=1}^{\infty} \Delta_{k_{i}}<\infty .
$$

This proves the desired result.

Lemma A.1 will be used to arrive at a contradiction. To obtain this contradiction, we first must show that for $k$ sufficiently large, Algorithm 4.2 produces a successful step.

Lemma A.2. Suppose there exists $\epsilon>0$ such that $\left\|\nabla m_{k}(0)\right\|_{\mathcal{Z}} \geq \epsilon$ for $k$ sufficiently large. Then, under Assumption 4.1, the ratios, $\left\{\varrho_{k}\right\}$, converge to one.

Proof. By Taylor's theorem, there exist $\theta_{k}$ and $\eta_{k}$ on the line segment between $s=0$ and $s=s_{k}$ such that

$$
\begin{aligned}
\operatorname{ared}_{k} & =\left\langle\nabla J\left(z_{k}\right), s_{k}\right\rangle_{\mathcal{Z}}+\frac{1}{2}\left\langle\nabla^{2} J\left(\theta_{k}\right) s_{k}, s_{k}\right\rangle_{\mathcal{Z}} \\
\operatorname{pred}_{k} & =\left\langle\nabla m_{k}(0), s_{k}\right\rangle_{\mathcal{Z}}+\frac{1}{2}\left\langle\nabla^{2} m_{k}\left(\eta_{k}\right) s_{k}, s_{k}\right\rangle_{\mathcal{Z}}
\end{aligned}
$$

These expansions and Assumption 4.1 imply

$$
\left|\operatorname{ared}_{k}-\operatorname{pred}_{k}\right| \leq \xi \Delta_{k-1} \Delta_{k}+\frac{1}{2}\left(\kappa_{1}+\kappa_{2}-1\right) \Delta_{k}^{2} .
$$

Furthermore, the fraction of Cauchy decrease condition, (4.2), and the assumption that $\left\|\nabla m_{k}(0)\right\|_{\mathcal{Z}} \geq \epsilon$ imply that for sufficiently large $k$,

$$
\operatorname{pred}_{k} \geq \kappa_{0}\left\|\nabla m_{k}(0)\right\|_{\mathcal{Z}} \min \left\{\Delta_{k}, \frac{\left\|\nabla m_{k}(0)\right\|_{\mathcal{Z}}}{\beta_{k}}\right\} \geq \kappa_{0} \epsilon \Delta_{k} .
$$

Combining these inequalities gives

$$
\left|\varrho_{k}-1\right| \leq \epsilon_{k}=\frac{\xi \Delta_{k-1}+\frac{1}{2}\left(\kappa_{1}+\kappa_{2}-1\right) \Delta_{k}}{\kappa_{0} \epsilon}
$$

for sufficiently large $k$. The sequence $\left\{\epsilon_{k}\right\}$ converges to zero by Lemma A.1, proving the result.

Remark A.3. For the CTR algorithm, $\Delta_{k-1}$ in the definition of $\epsilon_{k}$ is replaced by $\Delta_{k}$ and the same conclusion holds. In addition, the result of Lemma A.2 proves, for sufficiently large $k$, that CTR produces a successful step and increases the trust-region radius. This fact is sufficient to prove first-order convergence of CTR.

In addition to achieving a successful step, we must prove that Algorithm 4.2 increases the trust-region radius. This result is proved in a similar fashion to Lemma A.2.

Lemma A.4. Suppose there exists $\epsilon>0$ such that $\left\|\nabla m_{k}(0)\right\|_{\mathcal{Z}} \geq \epsilon$ for $k$ suffciently large. Then, under Assumption 4.1, the ratios, $\left\{\tilde{\varrho}_{k+1}\right\}$, converge to one.

Proof. By Taylor's theorem, there exists $\eta_{k+1}$ on the line segment between $s=0$ and $s=-s_{k}$ such that

$$
m_{k+1}\left(-s_{k}\right)-m_{k+1}(0)=-\left\langle\nabla m_{k+1}(0), s_{k}\right\rangle_{\mathcal{Z}}+\frac{1}{2}\left\langle\nabla^{2} m_{k+1}\left(\eta_{k+1}\right) s_{k}, s_{k}\right\rangle_{\mathcal{Z}} .
$$

This equality, the expansion of $\operatorname{pred}_{k}$ in the proof of Lemma A.2, and Assumption 4.1 imply

$$
\left|\operatorname{pred}_{k}-\left(m_{k+1}\left(-s_{k}\right)-m_{k+1}(0)\right)\right| \leq\left\|\nabla m_{k+1}(0)-\nabla m_{k}(0)\right\|_{\mathcal{Z}} \Delta_{k}+\left(\kappa_{2}-1\right) \Delta_{k}^{2} .
$$


To bound this further, notice that

$$
\begin{gathered}
\left\|\nabla m_{k+1}(0)-\nabla m_{k}(0)\right\|_{\mathcal{Z}} \leq\left\|\nabla m_{k+1}(0)-\nabla J\left(z_{k}+s_{k}\right)\right\|_{\mathcal{Z}}+\| \nabla J\left(z_{k}+s_{k}\right) \\
-\nabla J\left(z_{k}\right)\left\|_{\mathcal{Z}}+\right\| \nabla J\left(z_{k}\right)-\nabla m_{k}(0) \|_{\mathcal{Z}}
\end{gathered}
$$

The first and third expressions on the right-hand side of (A.1) are bounded by using (4.3), and the second expression is bounded using the differentiability of $J$, namely,

$$
\left\|\nabla J\left(z_{k}+s_{k}\right)-\nabla J\left(z_{k}\right)\right\|_{\mathcal{Z}}=\left\|\int_{0}^{1} \nabla^{2} J\left(z_{k}+t s_{k}\right) s_{k} \mathrm{~d} t\right\|_{\mathcal{Z}} \leq \kappa_{1} \Delta_{k} .
$$

This proves that

$$
\left|\operatorname{pred}_{k}-\left(m_{k+1}\left(-s_{k}\right)-m_{k+1}(0)\right)\right| \leq\left(\xi \Delta_{k}+\xi \Delta_{k-1}+\kappa_{1} \Delta_{k}\right) \Delta_{k}+\left(\kappa_{2}-1\right) \Delta_{k}^{2},
$$

which implies the bounds

$$
\operatorname{pred}_{k}-\tilde{\epsilon}_{k} \Delta_{k} \leq m_{k+1}\left(-s_{k}\right)-m_{k+1}(0) \leq \operatorname{pred}_{k}+\tilde{\epsilon}_{k} \Delta_{k}
$$

with $\tilde{\epsilon}_{k}=\left(\xi \Delta_{k}+\xi \Delta_{k-1}+\kappa_{1} \Delta_{k}+\left(\kappa_{2}-1\right) \Delta_{k}\right)$. The fraction of Cauchy decrease condition and the assumption that $\left\|\nabla m_{k}(0)\right\|_{\mathcal{Z}} \geq \epsilon$ imply

$$
\left(m_{k+1}\left(-s_{k}\right)-m_{k+1}(0)\right) \geq\left(\kappa_{0} \epsilon-\tilde{\epsilon}_{k}\right) \Delta_{k}
$$

Since $\tilde{\epsilon}_{k}$ converges to zero by Lemma A.1 and $\Delta_{k}>0$ for all $k$, the right-hand side of (A.2) is positive for sufficiently large $k$. Following the proof of Lemma A.2, these bounds and the fraction of Cauchy decrease condition (4.2) imply

$$
\begin{aligned}
\left|\varrho_{k+1}-1\right| & \leq\left|\frac{\operatorname{ared}_{k}-\operatorname{pred}_{k}}{m_{k+1}\left(-s_{k}\right)-m_{k+1}(0)}\right|+\left|\frac{\operatorname{pred}_{k}-\left(m_{k+1}\left(-s_{k}\right)-m_{k+1}(0)\right)}{m_{k+1}\left(-s_{k}\right)-m_{k+1}(0)}\right| \\
& \leq \frac{\epsilon_{k}+\tilde{\epsilon}_{k}}{\kappa_{0} \epsilon-\tilde{\epsilon_{k}}} \rightarrow 0 \quad \text { as } \quad k \rightarrow \infty .
\end{aligned}
$$

This proves the desired result.

Combining these results gives the desired result of the section: Algorithm 4.2 converges to a first-order critical point.

Theorem A.5. Suppose Assumptions 4.1 hold. Then

$$
\liminf _{k \rightarrow \infty}\left\|\nabla m_{k}(0)\right\|_{\mathcal{Z}}=\liminf _{k \rightarrow \infty}\left\|\nabla J\left(z_{k}\right)\right\|_{\mathcal{Z}}=0
$$

Proof. For contradiction, suppose there exists $\epsilon>0$ such that $\left\|\nabla m_{k}(0)\right\|_{\mathcal{Z}} \geq \epsilon$. By Lemma A.2, for $k$ sufficiently large there is a successful step $s_{k}$ since $\varrho_{k}$ converges to one. By Lemma A.4, for $k$ sufficiently large the trust-region radius must be increased since $\tilde{\varrho}_{k+1}$ converges to one. This fact contradicts the result of Lemma A.1.

Copyright (c) by SIAM. Unauthorized reproduction of this article is prohibited. 


\section{REFERENCES}

[1] F. Abergel and R. Temam, On some control problems in fluid mechanics, Theoret. Comput. Fluid Dynam., 1 (1990), pp. 303-325.

[2] N. Alexandrov, J. E. Dennis, Jr., R. M. Lewis, and V. Torczon, A trust region framework for managing the use of approximation models in optim., Structural Optim., 15 (1998), pp. 16-23.

[3] I. BABušKa, F. Nobile, And R. Tempone, A stochastic collocation method for elliptic partial differential equations with random input data, SIAM Rev., 52 (2010), pp. 317-355.

[4] I. BABUŠKA, R. Tempone, AND G. E. Zouraris, Galerkin finite element approximations of stochastic elliptic partial differential equations, SIAM J. Numer. Anal., 42 (2004), pp. 800-825.

[5] I. BabušKa, R. Tempone, And G. E. Zouraris, Solving elliptic boundary value problems with uncertain coefficients by the finite element method: the stochastic formulation, Comput. Methods Appl. Mech. Engrg., 194 (2005), pp. 1251-1294.

[6] V. Barthelmann, E. Novak, And K. Ritter, High dimensional polynomial interpolation on sparse grids, Adv. Comput. Math., 12 (2000), pp. 273-288.

[7] F. Bastin, C. Cirillo, and Ph. L. Toint, An adaptive Monte Carlo algorithm for computing mixed logit estimators, Comput. Manag. Sci., 3 (2006), pp. 55-79.

[8] F. Bastin, V. Malmedy, M. Mouffe, Ph. L. Toint, and D. Tomanos, A retrospective trust-region method for unconstrained optimization, Math. Program., 123 (2010), pp. 395-418.

[9] J. D. Benamou And B. Desprès, A domain decomposition method for the Helmholtz equation and related optimal control problems, J. Comput. Phys., 136 (1997), pp. 68-82.

[10] P. Bochev, H. C. Edwards, R. C. Kirby, K. Peterson, and D. Ridzal, Solving PDEs with Intrepid, Scientific Program., 20(2) (2012), pp. 151-181.

[11] A. Bonzì, Multigrid and sparse-grid schemes for elliptic control problems with random coeffcients, Comput. Vis. Sci., 13 (2010), pp. 153-160.

[12] A. Borzì, V. Schulz, C. Schillings, And G. von Winckel, On the treatment of distributed uncertainties in PDE constrained optimization, GAMM Mitt., 33 (2010), pp. 230-246.

[13] A. Borzì And G. VON Winckel, Multigrid methods and sparse-grid collocation techniques for parabolic optimal control problems with random coefficients, SIAM J. Sci. Comput., 31 (2009), pp. 2172-2192.

[14] H.-J. Bungartz And M. Griebel, Sparse grids, Acta Numer., 13 (2004), pp. 147-269.

[15] J. A. Burns, T. Lin, and L. G. Stanley, A Petrov Galerkin finite-element method for interface problems arising in sensitivity computations, Comput. Math. Appl., 49 (2005), pp. 1889-1903.

[16] Y. CAO, M. Y. Hussaini, AND H. YANG, Numerical optimization of radiated engine noise with uncertain wavenumbers, Internat. J. Numer. Anal. Model., 4 (2007), pp. 392-401.

[17] R. G. CARTER, Numerical experience with a class of algorithms for nonlinear optimization using inexact function and gradient information, SIAM J. Sci. Comput., 14 (1993), pp. 368-388.

[18] B. Cengiz, On the duals of Lebesgue-Bochner $L^{p}$ spaces, Proc. Amer. Math. Soc., 114 (1992), pp. 923-926.

[19] S. Chaillat and G. Biros, FaIMS: A fast algorithm for the inverse medium problem with multiple frequencies and multiple sources for the scalar Helmholtz equation, J. Comput. Phys., 231 (2012), pp. 4403-4421.

[20] A. Chrifa, A. Cohen, R. DeVore, and C. Schwab, Sparse adaptive Taylor approximation algorithms for parametric and stochastic elliptic pdes, ESAIM Math. Model. Numeri. Anal., 47 (2013), pp. 253-280.

[21] A. R. Conn, N. I. M. Gould, And Ph. L. Toint, Trust-Region Methods, SIAM, Philadelphia, 2000.

[22] S. Conti, H. Held, M. Pach, M. Rumpf, and R. Schultz, Shape optimization under uncertainty: A stochastic programming perspective, SIAM J. Optim., 19 (2008), pp. 1610-1632.

[23] S. Conti, H. Held, M. Pach, M. Rumpf, and R. Schultz, Risk averse shape optimization, SIAM J. Control Optim., 49 (2011), pp. 927-947.

[24] A. Debussche, M. Fuhrman, and G. Tessitore, Optimal control of a stochastic heat equation with boundary-noise and boundary control, ESAIM Control, Optim. Calc. Var., 13 (2007), pp. 178-205.

[25] T. Gerstner and M. Griebel, Numerical integration using sparse grids, Numer. Algorithms, 18 (1998), pp. 209-232. 
[26] T. Gerstner and M. Griebel, Dimension-adaptive tensor-product quadrature, Computing, 71 (2003), pp. 65-87.

[27] V. Girault And P.-A. Raviart, Finite Element Approximation of the Navier-Stokes Equations, Lecture Notes in Math. 749, Springer-Verlag, Berlin, 1979.

[28] M. Griebel And S. KnAPeK, Optimized general sparse grid approximation spaces for operator equations, Math. Comp., 78 (2009), pp. 2223-2257.

[29] M. D. Gunzburger, Perspectives in Flow Control and Optimization, SIAM, Philadelphia, 2003.

[30] M. D. Gunzburger, H.-C. Lee, and J. Lee, Error estimates of stochastic optimal neumann boundary control problems, SIAM J. Numer. Anal., 49 (2011), pp. 1532-1552.

[31] J. M. HARris, The Influence of Random Media on the Propagation and Depolarization of Electromagnetic Waves, Ph.D. thesis, California Institute of Technology, Pasadena, 1980.

[32] M. Heinkenschloss, Formulation and analysis of a sequential quadratic programming method for the optimal boundary control of Navier-Stokes flow, in Optimal Control: Theory, Algorithms, and Applications, W. W. Hager and P. M. Pardalos, eds., Kluwer Academic, Publishers, Norwell, MA, 1998, pp. 178-203.

[33] M. Heinkenschloss And L. N. Vicente, Analysis of inexact trust-region SQP algorithms, SIAM J. Optim., 12 (2001), pp. 283-302.

[34] M. A. Heroux, R. A. Bartlett, V. E. Howle, R. J. Hoekstra, J. J. Hu, T. G. Kolda, R. B. Lehoucq, K. R. Long, R. P. Pawlowski, E. T. Phipps, A. G. Salinger, H. K. Thornquist, R. S. Tuminaro, J. M. Willenbring, A. Williams, and K. S. Stanley, An overview of the Trilinos project, ACM Trans. Math. Softw., 31 (2005), pp. 397-423.

[35] M. A. Heroux and J. M. Willenbring, A new overview of the Trilinos project, Sci. Program., to appear.

[36] M. Hinze, R. Pinnau, M. Ulbrich, and S. Ulbrich, Optimization with Partial Differential Equations, Math. Model., Theory Appl. 23, Springer-Verlag, Berlin, 2009.

[37] G. E. Karniadakis, C.-H. Su, D. Xiu, D. Lucor, C. Schwab, and R. A. Todor, Generalized Polynomial Chaos Solution for Differential Equations with Random Inputs, Tech. report 2005-01, Seminar for Applied Mathematics, ETH Zurich, 2005.

[38] D. P. KourI, Optimization Governed by Stochastic Partial Differential Equations, M.S. thesis, Department of Computational and Applied Mathematics, Rice University, Houston, TX, 2010.

[39] D. P. Kouri, An Approach for the Adaptive Solution of Optimization Problems Governed by Partial Differential Equations with Uncertain Coefficients, Department of Computational and Applied Mathematics, Rice University, Houston, TX, PhD thesis, 2012.

[40] A. Kunoth and Ch. Schwab, Analytic Regularity and GPC Approximation for Control Problems Constrained by Linear Parametric Elliptic and Parabolic PDEs, SAM research report 2011-54, Seminar für Angewandte Mathematik, ETH Zürich, 2011.

[41] P. Larkin and M. Whalen, Direct, Near Field Acoustic Testing, SAE technical paper 199901-5553, SAE International, Warrendale, PA, 1999.

[42] G. D. Manolis and A. C. Bagtzoglou, A numerical comparative study of wave propagation in inhomogeneous and random media, Comput. Mech., 10 (1992), pp. 397-413.

[43] J. J. MoRÉ, Recent developments in algorithms and software for trust region methods, in Mathematical Programming, The State of the Art, A. Bachem, M. Grötschel, and B. Korte, eds., Springer-Verlag, Berlin, 1983, pp. 258-287.

[44] Jerome A. Neubert and John L. Lumley, Derivation of the stochastic helmholtz equation for sound propagation in a turbulent fluid, J. Acoust. Soc. Amer., 48 (1970), pp. $1212-1218$.

[45] F. Nobile, R. Tempone, and C. G. Webster, The Analysis of a Sparse Grid Stochastic Collocation Method for Partial Differential Equations with High-Dimensional Random Input Data, Tech. report SAND2007-8093, Sandia National Laboratories, 2007.

[46] F. Nobile, R. Tempone, And C. G. Webster, An nisotropic sparse grid stochastic collocation method for partial differential equations with random input data, SIAM J. Numer. Anal., 46 (2008), pp. 2411-2442.

[47] F. Nobile, R. Tempone, And C. G. Webster, A sparse grid stochastic collocation method for partial differential equations with random input data, SIAM J. Numer. Anal., 46 (2008), pp. 2309-2345.

[48] E. NovaK AND K. RItTeR, High-dimensional integration of smooth functions over cubes, Numer. Math., 75 (1996), pp. 79-97.

[49] E. Novak And K. Ritter, Simple cubature formulas with high polynomial exactness, Constr. Approx., 15 (1999), pp. 499-522. 
[50] E. NovaK AND H. Woźniakowski, Tractability of multivariate problems. Volume II: Standard Information for Functionals, EMS Tracts in Math. 12, European Mathematical Society, Zürich, 2010.

[51] K. Petras, On the Smolyak cubature error for analytic functions, Adv. Comput. Math., 12 (2000), pp. 71-93.

[52] K. PETRAs, Smolyak cubature of given polynomial degree with few nodes for increasing dimension, Numer. Math., 93 (2003), pp. 729-753.

[53] G. Da Prato and A. Debussche, Control of the stochastic burgers model of turbulence, SIAM J. Control Optim., 37 (1999), pp. 1123-1149.

[54] A. Quarteroni And A. Valli, Domain Decomposition Methods for Partial Differential Equations, Oxford University Press, Oxford, UK, 1999.

[55] C. Schillings, Optimal Aerodynamic Design Under Uncertainties, Ph.D. thesis, Fb-IV, Mathematik, Universität Trier, Germany, 2010.

[56] V. Schulz and C. Schillings, On the nature and treatment of uncertainties in aerodynamic design, AIAA J., 47 (2009), pp. 646-654.

[57] A. Shapiro, D. Dentcheva, And A. Ruszczynski, Lectures on Stochastic Programming: Modeling and Theory, SIAM, Philadelphia, 2009.

[58] S. A. SmolJak, Quadrature and interpolation formulae on tensor products of certain function classes, Soviet Math. Dokl., 4 (1963), pp. 240-243.

[59] M.L. Stein, Interpolation of Spatial Data: Some Theory for Kriging, Springer-Verlag, New York, 1999.

[60] H. Tiesler, R. M. Kirby, D. Xiu, and T. Preusser, Stochastic collocation for optimal control problems with stochastic PDE constraints, SIAM J. Control Optim., 50 (2012), pp. 26592682.

[61] F. TröLtzsch, Optimal Control of Partial Differential Equations: Theory, Methods and Applications, Grad. Stud. Math. 112, AMS, Providence, RI, 2010.

[62] S. Volkwein, Mesh-Independence of an Augmented Lagrangian-SQP Method in Hilbert Spaces and Control Problems for the Burgers Equation, Ph.D. thesis, Fachbereich Mathematik, Technische Universität Berlin, Germany, 1997; also available online from www.unigraz.at/imawww/volkwein/diss.ps.

[63] S. VolKWeIn, Application of the augmented Lagrangian-SQP method to optimal control problems for the stationary Burgers equation, Comput. Optim. Appl., 16 (2000), pp. 57-81.

[64] G. W. Wasilkowski and H. WoźNIAKOWski, Explicit cost bounds of algorithms for multivariate tensor product problems, J. Complexity, 11 (1995), pp. 1-56.

[65] C. Webster, Sparse Grid Stochastic Collocation Techniques for the Numerical Solution of Partial Differential Equations with Random Input Data, Ph.D. thesis, Department of Mathematics and School of Computational Science, Florida State University, Tallahassee, 2007.

[66] D. Xiu and J. S. Hesthaven, High-order collocation methods for differential equations with random inputs, SIAM J. Sci. Comput., 27 (2005), pp. 1118-1139.

[67] D. Xiu And G. E. Karniadakis, Modeling uncertainty in flow simulations via generalized polynomial chaos, J. Comput. Phys., 187 (2003), pp. 137-167.

[68] D. XiU And J. Shen, An efficient spectral method for acoustic scattering from rough surfaces, Commun. Comput. Phys., 2 (2007), pp. 54-72.

[69] K. Yosida, Functional Analysis, 6th ed., Springer-Verlag, Berlin, 1980.

[70] E. ZeIdLer, Nonlinear Functional Analysis and its Applications II/A: Linear Monotone Operators, Springer-Verlag, Berlin, 1990.

[71] T. ZHou, Stochastic Galerkin methods for elliptic interface problems with random input, J. Comput. Appl. Math., 236 (2011), pp. 782-792.

[72] J. C. Ziems And S. Ulbrich, Adaptive Multilevel Generalized SQP-Methods for PDEConstrained Optimization, Tech. report, Fachbereich Mathematik, Technische Universität Darmstadt, Germany, 2011. 